\title{
Correlated transport and non-Fermi-liquid behavior in single-wall carbon nanotubes
}

\author{
Reinhold Egger ${ }^{1}$ and Alexander O. Gogolin ${ }^{2}$ \\ ${ }^{1}$ Fakultät für Physik, Albert-Ludwigs-Universität, Hermann-Herder-Straße 3, D-79104 Freiburg, Germany \\ ${ }^{2}$ Department of Mathematics, Imperial College, 180 Queen's Gate, London SW7 2BZ, United Kingdom
}

(Date: September 13, 2018)

We derive the effective low-energy theory for single-wall carbon nanotubes including the Coulomb interactions among electrons. The generic model found here consists of two spin$\frac{1}{2}$ fermion chains which are coupled by the interaction. We analyze the theory using bosonization, renormalization-group techniques, and Majorana refermionization. Several experimentally relevant consequences of the breakdown of Fermi liquid theory observed here are discussed in detail, e.g., magnetic instabilities, anomalous conductance laws, and impurity screening profiles.

PACS numbers: 71.10.Pm, 71.20.Tx, 72.80.Rj

\section{INTRODUCTION}

The fascinating electronic and mechanical properties of carbon nanotubes have recently attracted a lot of attention [1]. Nanotubes are tubular nanoscale objects which can be thought of as graphite sheets wrapped into a cylinder. Shortly after their discovery during the carbon-arc fullerene production [2], theorists have made a surprising prediction linking structural to electronic properties [3 6]. Specifically, the arrangement of carbon atoms on the tube surface is determined by the integer indices $0 \leq m \leq n$ of the wrapping superlattice vector $\boldsymbol{T}=n \boldsymbol{a}_{1}+m \boldsymbol{a}_{2}$, where $\boldsymbol{a}_{1}$ and $\boldsymbol{a}_{2}$ are the primitive Bravais translation vectors of the honeycomb lattice. Depending on the choice of $n$ and $m$, a $(n, m)$ nanotube should then be either a metal, a narrow-gap semiconductor, or an insulator. This theoretical prediction has been amply confirmed in recent experiments [711].

Nanotubes can be fabricated using either the carbonarc method 2,12 or a novel laser ablation technique [10], where Co- or Ni-doped graphite targets are laservaporized. The carbon-arc process usually yields multiwall nanotubes (MWNT) composed of several concentric graphite sheets. One can then attach metallic leads to such a MWNT and measure, e.g., the magnetoconductance. The MWNT experiment by Langer et al. [13] shows typical signs of a weakly disordered mesoscopic system, such as universal conductance fluctuations or weak localization phenomena. On the other hand, the laser ablation method allows for the controlled fabrication of single-wall nanotubes (SWNT). Rather large quantities of metallic $(n, n)$ "armchair" nanotubes with $n=10$ can be obtained using this technique [10]. In most cases, the SWNTs spontaneously crystallize in triangular-packed ropes containing $\approx 20$ to 100 SWNTs, to which one can again attach leads and perform transport measurements. The experimental results of Fischer et al. 14 have shown a linear temperature dependence of the resistance above a (non-universal) crossover temperature, with an increase of the resistance at lower temperatures. In addition, the experiments of Bockrath et al. [15] showed Coulomb charging effects [16] in such a rope.

One can in fact also produce single SWNTs using the laser ablation process. From a fundamental (and theoretical) point of view, a single SWNT is an intriguing system, since Coulomb interactions induce a breakdown of Fermi liquid theory [17 in any one-dimensional (1D) metal. Astonishingly, Tans et al. [18] were able to attach contacts to a single $3 \mu \mathrm{m}$ long $(10,10)$ armchair SWNT. The transport measurements of Ref. [18] were dominated by charging effects due to large contact resistances [around $500 \mathrm{k} \Omega$ ] between the leads and the SWNT. By finding a way to decrease the contact resistances one could circumvent charging effects, thereby allowing one to study the peculiar properties of a 1D conductor. Nanotubes are potentially much more stable $1 \mathrm{D}$ conductors than conventional systems such as chain molecules [19], edge states in the (fractional) quantum Hall effect [20], or quantum wires in semiconductor heterostructures [21]. In these systems, non-Fermi liquid behavior has been notoriously difficult to establish experimentally.

As has been demonstrated in Ref. [18], a metallic SWNT constitutes a perfect experimental realization of a 1D conductor. Interacting 1D electrons exhibit Luttinger liquid rather than Fermi liquid behavior characterized by, e.g., the absence of Landau quasi-particles, spin-charge separation, suppression of the electron tunneling density of states, and interaction-dependent power laws for transport quantities. For detailed accounts of the physics of Luttinger liquids, see, e.g., Refs. 222 23. In this paper we describe the nature of the non-Fermi-liquid state in a SWNT by deriving and analyzing the effective low-energy theory of carbon nanotubes. This state should be observable as soon as charging effects are overcome by lower contact resistances. A brief and incomplete account of our results has been given in Ref. [24].

The role of Coulomb interactions is most pronounced if the nanotube is metallic. What are the conditions for metallicity? A necessary condition arises because the Fermi vector should obey the transverse quantization condition $\boldsymbol{T} \boldsymbol{k}=2 \pi I$ for an integer $I$. The first Brillouin 
zone of the honeycomb lattice is a hexagon, and bandstructure calculations 25,26] show that the only gapless points are the corner points of this hexagon. One then finds that there are exactly two independent Fermi points denoted by $K$ and $K^{\prime}$, with two linearly dispersing bands around each of these two points. Imposing the transverse quantization condition for the Fermi vector corresponding to $K$ (or $K^{\prime}$ ) implies $2 n+m=3 I$. If this condition is not fulfilled, the nanotube exhibits the "primary" band gap $\Delta E=2 v / 3 R \approx 1 \mathrm{eV}$, where $v$ is the Fermi velocity and $R$ the radius. We mention in passing that a nanotube without primary gap has a finite Fermi momentum only if $n-m=3 d I$, where $d$ is the highest common divisor of $n$ and $m$ [27].

Even if the necessary condition $2 n+m=3 I$ is fulfilled, the rearrangement of local bonds due to the curvature of the cylinder can introduce a "secondary" gap, $\Delta E \approx 10$ meV, which implies narrow-gap semiconducting behavior. For very small tube diameter, e.g., for a $(6,0)$ tube, due to the strong curvature-induced hybridization of $\sigma$ and $\pi$ orbitals, this effect can be quite pronounced [28. In the case of armchair nanotubes $(n=m)$, however, the formation of a secondary gap is prevented by the high symmetry, and therefore a $(n, n)$ SWNT stays metallic for all $n$. The highly symmetric structure of $(n, n)$ [and $(n, 0)$ "zig-zag"] nanotubes also prohibits chirality, i.e., the carbon atoms close around the waist of the nanotube. In contrast, all other nanotubes show chiral behavior [29].

One-dimensional metals are expected to exhibit a Peierls instability due to the spontaneous formation of a lattice distortion. It has been argued in Refs. [3,6] that this instability should not be of any practical importance in nanotubes. Mean-field estimates of the Peierls transition temperature yield very low values $(\approx 1 \mathrm{~K})$, and fluctuations will then tend to wash out the instability even further. We therefore neglect coupling to lattice distortions in the following.

So far we have discussed the case of a perfectly clean SWNT. Nevertheless, there are various sources for impurities, e.g., structural imperfections of the nanotube (like substitutional atoms), charge defects in the substrate, topological defects, or twists. Topological defects are dislocations (kinks) that locally change the superlattice vector $\boldsymbol{T}$ by replacing one of the hexagons in the graphite network by a pentagon or a heptagon. Such a change typically introduces a metal-insulator interface. By combining a kink and its antikink (dislocation pair), it is possible to form local metal-insulator-metal junctions [30]. Transport is then dominated by tunnel events through this barrier. A less dramatic but probably more widespread source for backscattering is given by twists of the nanotube. These twists can originate from the momentary position of the SWNT when deposited onto the leads, or from thermal fluctuation modes 31.

Generally, especially in the presence of impurities, pronounced effects of the Coulomb interaction on transport in 1D metals can be expected [32]. As shown in Ref. [33], at very low energy scales transport is fully blocked by a single arbitrarily weak impurity. The conductance then vanishes as a function of temperature and/or voltage with anomalous interaction-dependent power laws. This effect can be understood in terms of the Friedel oscillation 34.35 building up around a scatterer in a 1D metal. The oscillatory charge screening cloud displays an algebraically slow decay away from the impurity and thereby causes an important additional backscattering contribution [36]. This crucial effect cannot be captured by linear screening [37]. The dielectric function approach to impurity screening in SWNTs [38 is therefore practically useless in a determination of the Friedel oscillation.

Below we focus on a single armchair SWNT, where interaction effects are very pronounced and direct experimental tests of the theory are within reach. However, as long as the above-mentioned band gaps are negligible, e.g., for sufficiently high temperatures, our effective lowenergy Hamiltonian applies to any SWNT, even a chiral one. The only change arising for $n \neq m$ affects the precise value of the interaction parameters introduced below. This remarkable circumstance is due to a $U(1)$ symmetry of the graphite dispersion relation present close to the Fermi surface. Finally, we mention that by adding a bulk mass term, the properties of semiconducting or insulating SWNTs could be analyzed within the same framework [39].

The structure of this paper is as follows. In Sec. II the effective low-energy description of SWNTs is derived. The resulting fermionic model is bosonized in Sec. III. A renormalization group analysis is carried out in Sec. IV and supplemented by the Majorana refermionization approach described in Sec. V. Experimentally relevant susceptibilities and correlation functions are discussed in Sec. VI. In Sec. VII, we study transport through the nanotube, followed by a discussion of impurity screening in Sec. VIII. Finally, some concluding remarks can be found in Sec. IX. Throughout this paper, we put $\hbar=1$.

\section{EFFECTIVE LOW-ENERGY THEORY}

In this section we derive the effective low-energy description of a single-wall $(n, n)$ carbon nanotube. Our description applies at energy scales where the generic linear dispersion relation of a SWNT depicted in Fig. 1 is valid. For a $(10,10)$ nanotube, the theory therefore holds at room temperature and below. A similar approach has been devised previously for the uncorrelated case by Kane and Mele [31], building on earlier work 440] for graphite intercalation compounds. Here we extend their theory and include the Coulomb interactions between the electrons. Previously, this problem has only been investigated using the perturbative renormalization group (RG) for a weak short-range (Hubbard) interaction 
41,42. However, there is no external screening of the Coulomb interaction in the SWNT experiments of Ref. [18], and one has to take into account the long-ranged character of the Coulomb interaction potential (see also Ref. [43]). Since one might employ suitable screening backgates in future experiments, the general (short- or long-ranged) case of an arbitrary interaction potential is treated here. The method used below allows us to get insight into the physics of the strong-coupling regime emerging at low temperatures.

\section{A. Low-energy expansion}

The remarkable electronic properties of carbon nanotubes are due to the special bandstructure of the $\pi$ electrons in graphite. The simplest bandstructure calculation starts from a nearest-neighbor tight-binding Hamiltonian on the honeycomb lattice, which can be straightforwardly diagonalized [25]. Remarkably, the only gapless points of the resulting dispersion relation are the corner points of the hexagonal first Brillouin zone. Hence there are only two linearly independent Fermi points $\alpha \boldsymbol{K}$ with $\alpha= \pm$ instead of a continuous Fermi surface. Up to energy scales $\leq 1 \mathrm{eV}$, the dispersion relation around the Fermi points is, to a very good approximation, linear,

$$
E_{ \pm}(\boldsymbol{q}=\boldsymbol{k}-\boldsymbol{K})= \pm v|\boldsymbol{q}|
$$

with the same relation around the other Fermi point. The $+(-)$ sign corresponds to the conduction (valence) band, respectively. Obviously, close to the Fermi surface a $U(1)$ symmetry holds, since the direction of $\boldsymbol{q}$ does not matter in Eq. 2.1).

Since the basis of the honeycomb lattice contains two atoms, there are two sublattices $p= \pm$ shifted by the vector $\boldsymbol{d}=(0, d)$, with the nearest-neighbor C-C distance $d=a / \sqrt{3}=1.42 \AA$ (here $a$ denotes the honeycomb lattice constant). As a consequence, there are two degenerate Bloch states

$$
\varphi_{p \alpha}(\boldsymbol{r})=(2 \pi R)^{-1 / 2} \exp (-i \alpha \boldsymbol{K} \boldsymbol{r})
$$

at each Fermi point $\alpha= \pm$, where $\boldsymbol{r}=(x, y)$ lives on the sublattice $p$ under consideration. In Eq. (2.2), we have already anticipated the correct normalization for nanotubes. We follow Ref. [40] and choose the Bloch functions separately on each sublattice such that they vanish on the other. One can then expand the electron operator in terms of these Bloch waves ( $\sigma= \pm$ is the spin index),

$$
\Psi_{\sigma}(x, y)=\sum_{p \alpha} \varphi_{p \alpha}(x, y) F_{p \alpha \sigma}(x, y),
$$

which introduces slowly varying operators $F_{p \alpha \sigma}(x, y)$. The resulting second-quantized effective low-energy theory of a graphite sheet is given by the 2D massless Dirac Hamiltonian 40],

$$
H_{G}=-i v \sum_{p p^{\prime} \alpha \sigma} \int d \boldsymbol{r} F_{p \alpha \sigma}^{\dagger}(\boldsymbol{\tau} \nabla)_{p p^{\prime}} F_{p^{\prime} \alpha \sigma},
$$

in accordance with the spectrum (2.1). Here $\boldsymbol{\tau}=\left(\tau_{x}, \tau_{y}\right)$ are standard Pauli matrices.

Wrapping the graphite sheet leads to the generic bandstructure of a metallic SWNT shown in Fig. 1. For a $(n, n)$ armchair SWNT, the Fermi vector is $\boldsymbol{K}=\left(k_{F}, 0\right)$ with $k_{F}=4 \pi / 3 a$. We take the $x$-axis along the tube direction, and the circumferential variable is $0<y<2 \pi R$. The armchair SWNT radius is $R=\sqrt{3} n a / 2 \pi$, which yields $R=1.38 \mathrm{~nm}$ for a $(10,10)$ nanotube. Quantization of transverse motion now allows for a contribution $\exp (i m y / R)$ to the wavefunction. However, excitation of angular momentum states other than $m=0$ costs the energy $\approx 10 \mathrm{eV} / n$. In an effective low-energy theory, we may thus omit all transport bands except $m=0$ and hence arrive at a $1 \mathrm{D}$ situation with $k_{y}=0$. Since also $K_{y}=0$, the Bloch states (2.2) do not depend on the circumferential coordinate, and the corresponding wavefunctions are indeed stiff around the waist of the nanotube.

Instead of the low-energy expansion (2.3), the electron operator is now written as 31]

$$
\Psi_{\sigma}(x, y)=\sum_{p \alpha} \varphi_{p \alpha}(x, y) \psi_{p \alpha \sigma}(x),
$$

which introduces 1D fermion operators $\psi_{p \alpha \sigma}(x)$ depending only on the $x$ coordinate. They correspond to the $F_{p \alpha \sigma}$ for the graphite sheet. Neglecting Coulomb interactions for the moment, the Hamiltonian can be read off from Fig. 1,

$$
H_{0}=-v \sum_{p \alpha \sigma} p \int d x \psi_{p \alpha \sigma}^{\dagger} \partial_{x} \psi_{-p \alpha \sigma} .
$$

For this expression, we have chosen a preferred direction in Eq. (2.4). However, due to the mentioned $U(1)$ invariance, we can choose any direction without affecting the resulting low-energy Hamiltonian [we have explicitly checked that this holds even in the presence of interactions]. Switching from the sublattice $(p= \pm)$ description to the right- and left-movers $(r= \pm)$ indicated in Fig. 1 implies two copies $(\alpha= \pm)$ of massless 1D Dirac Hamiltonians. By using a suitable gate, one can experimentally tune the average charge density on the nanotube and hence adjust the Fermi energy. In contrast to the half-filled band $\left(E_{F}=0\right)$ encountered in $2 \mathrm{D}$ graphite sheets, one is normally off half-filling in SWNT experiments. Typically, the Fermi energy is displaced by about $300 \mathrm{meV}$ in the experiment of Ref. [18]. Finally, to describe semi-conducting or insulating SWNTs with a band gap $\Delta E$, a bulk mass term should be added 39,

$$
H^{\prime}=\frac{\Delta E}{2} \int d x \sum_{p \alpha \sigma} \psi_{p \alpha \sigma}^{\dagger} \psi_{-p \alpha \sigma} .
$$


We will put $\Delta E=0$ in the following, as is appropriate for armchair nanotubes.

\section{B. Coulomb interaction}

Let us now examine Coulomb interactions mediated by an arbitrary potential $U\left(\boldsymbol{r}-\boldsymbol{r}^{\prime}\right)$. The interaction is described by the Hamiltonian

$$
\begin{aligned}
H_{I}=\frac{1}{2} & \sum_{\sigma \sigma^{\prime}} \int d \boldsymbol{r} \int d \boldsymbol{r}^{\prime} \Psi_{\sigma}^{\dagger}(\boldsymbol{r}) \Psi_{\sigma^{\prime}}^{\dagger}\left(\boldsymbol{r}^{\prime}\right) \\
& \times U\left(\boldsymbol{r}-\boldsymbol{r}^{\prime}\right) \Psi_{\sigma^{\prime}}\left(\boldsymbol{r}^{\prime}\right) \Psi_{\sigma}(\boldsymbol{r}) .
\end{aligned}
$$

Herein we have assumed that the interaction is not sensitive to the electron spin $\sigma= \pm$. Bound electrons can be incorporated in terms of a background dielectric constant $\kappa$, but free charge carriers in nearby gates could lead to an effectively short-ranged potential.

For the experiments of Ref. [18], one has an externally unscreened Coulomb interaction,

$$
U\left(\boldsymbol{r}-\boldsymbol{r}^{\prime}\right)=\frac{e^{2} / \kappa}{\sqrt{\left(x-x^{\prime}\right)^{2}+4 R^{2} \sin ^{2}\left[\left(y-y^{\prime}\right) / 2 R\right]+a_{z}^{2}}},
$$

where $a_{z} \simeq 3 a_{B} \approx a$ [with the Bohr radius $a_{B}=$ $\left.\hbar^{2} / m e^{2}=0.529 \AA\right]$ denotes the average distance between a $2 p_{z}$ electron and the nucleus, i.e., the "thickness" of the graphite sheet. The dielectric constant in Eq. (2.9) can be estimated from the following elementary consideration. In a strictly $1 \mathrm{D}$ system of length $L$ with interaction potential $u\left(x-x^{\prime}\right)=e^{2} / \kappa\left|x-x^{\prime}\right|$, the charging energy is

$$
\begin{aligned}
E_{c} & =\frac{1}{2 L^{2}} \int_{0}^{L} d x d x^{\prime} u\left(x-x^{\prime}\right) \\
& \simeq e^{2} \ln (L / R) / \kappa L .
\end{aligned}
$$

The experimental value $E_{c}=2.6 \mathrm{meV}$ [18 for $L=3 \mu \mathrm{m}$ leads to $\kappa \approx 1.4$. The theoretical estimate $\kappa \approx 2.4$ for bulk graphite 44 is of the same order of magnitude. Our estimate also includes the effect of the insulating substrate.

The interaction (2.8) can now be reduced to a $1 \mathrm{D}$ interaction by inserting the expansion (2.5) for the electron field operator. The result is

$$
\begin{aligned}
H_{I} & =\frac{1}{2} \sum_{p p^{\prime} \sigma \sigma^{\prime}} \sum_{\alpha_{1} \alpha_{2} \alpha_{3} \alpha_{4}} \int d x d x^{\prime} V_{\left\{\alpha_{i}\right\}}^{p p^{\prime}}\left(x-x^{\prime}\right) \\
& \times \psi_{p \alpha_{1} \sigma}^{\dagger}(x) \psi_{p^{\prime} \alpha_{2} \sigma^{\prime}}^{\dagger}\left(x^{\prime}\right) \psi_{p^{\prime} \alpha_{3} \sigma^{\prime}}\left(x^{\prime}\right) \psi_{p \alpha_{4} \sigma}(x)
\end{aligned}
$$

with the $1 \mathrm{D}$ interaction potentials

$$
\begin{gathered}
V_{\left\{\alpha_{i}\right\}}^{p p^{\prime}}\left(x-x^{\prime}\right)=\int_{0}^{2 \pi R} d y d y^{\prime} \varphi_{p \alpha_{1}}^{*}(x, y) \varphi_{p^{\prime} \alpha_{2}}^{*}\left(x^{\prime}, y^{\prime}\right) \\
\times U\left(x-x^{\prime}, y-y^{\prime}+p d \delta_{p,-p^{\prime}}\right) \varphi_{p^{\prime} \alpha_{3}}\left(x^{\prime}, y^{\prime}\right) \varphi_{p \alpha_{4}}(x, y) .
\end{gathered}
$$

These potentials only depend on $x-x^{\prime}$ and on the 1D fermion quantum numbers. For interactions involving different sublattices for $\boldsymbol{r}$ and $\boldsymbol{r}^{\prime}$ in Eq. (2.8), i.e., $p \neq p^{\prime}$, one needs to take into account the shift vector $\boldsymbol{d}=(0, d)$, see Eq. 2.11).

To simplify the resulting 1D interaction (2.10), we now exploit momentum conservation. Since an additional gate voltage tunes the average electron density on the SWNT, we assume that no lattice instabilities due to Umklapp scattering are present, i.e., one stays off halffilling. This assumption does not limit the applicability of our approach, but simplifies matters considerably. For a recent study of Umklapp processes in SWNTs, see Ref. 42]. We then employ the Fermi point quantum numbers $\alpha_{i}$ to classify the allowed processes, see Fig. 2. First, there are "forward scattering" $(\alpha \mathrm{FS})$ processes, where $\alpha_{1}=\alpha_{4}$ and $\alpha_{2}=\alpha_{3}$. Second, we have "backscattering" ( $\alpha \mathrm{BS})$, with $\alpha_{1}=-\alpha_{2}=\alpha_{3}=-\alpha_{4}$. Notice that the above classification differs from the conventional one for the standard two-chain problem [23]. The latter is based on the right- and left-moving indices $(r= \pm$ or $p= \pm$ ) rather than on the different Fermi points of the nanotube $(\alpha= \pm)$.

\section{Forward scattering}

Let us start with $\alpha \mathrm{FS}$, where $\alpha_{1}=\alpha_{4}$ and $\alpha_{2}=\alpha_{3}$. The corresponding diagram is shown in Fig. 2. We first define the potential

$$
V_{0}\left(x-x^{\prime}\right)=\int_{0}^{2 \pi R} \frac{d y}{2 \pi R} \int_{0}^{2 \pi R} \frac{d y^{\prime}}{2 \pi R} U\left(\boldsymbol{r}-\boldsymbol{r}^{\prime}\right) .
$$

This gives from Eqs. (2.11) and (2.2) the $\left\{\alpha_{i}\right\}$ independent forward scattering interaction potential

$$
V_{\alpha \mathrm{FS}}^{p p^{\prime}}(x)=V_{0}(x)+\delta_{p,-p^{\prime}} \delta V_{p}(x),
$$

with the correction term

$$
\delta V_{p}(x)=\int_{0}^{2 \pi R} \frac{d y d y^{\prime}}{(2 \pi R)^{2}}\left[U\left(x, y-y^{\prime}+p d\right)-U\left(x, y-y^{\prime}\right)\right]
$$

which is only present if $\boldsymbol{r}$ and $\boldsymbol{r}^{\prime}$ are located on different sublattices. Thereby important information about the discrete nature of the graphite network is retained despite the low-energy continuum approximation employed in our formulation. The correction $\delta V_{p}$ is a direct measure of the difference between intra- and inter-sublattice interactions. Because of the periodicity of the $y$-integrals, Taylor expanding Eq. (2.14) in powers of $d$ implies that the correction $\delta V_{p}(x)=0$. Since $V_{0}(x)$ treats both sub-

(2.11) lattices on equal footing, the resulting $\alpha \mathrm{FS}$ interaction couples only the total electron densities, 


$$
H_{\alpha \mathrm{FS}}^{(0)}=\frac{1}{2} \int d x d x^{\prime} \rho(x) V_{0}\left(x-x^{\prime}\right) \rho\left(x^{\prime}\right),
$$

where the $1 \mathrm{D}$ density is defined as

$$
\rho(x)=\sum_{p \alpha \sigma} \psi_{p \alpha \sigma}^{\dagger}(x) \psi_{p \alpha \sigma}(x) .
$$

This density does not contain "fast" terms arising from a mixture of the different $(p \alpha \sigma)$-type fermions. Such terms turn out to be crucial for an understanding of the Friedel oscillation in Sec.VIII.

For the unscreened Coulomb interaction (2.9), the potential (2.12) becomes

$$
V_{0}(x)=\frac{2 e^{2}}{\kappa \pi \sqrt{a_{z}^{2}+x^{2}+4 R^{2}}} K\left(\frac{2 R}{\sqrt{a_{z}^{2}+x^{2}+4 R^{2}}}\right),
$$

with the complete elliptic integral $K(z)$ of the first kind [45]. For $x \gg R$, one finds $V_{0}(x) \sim 1 / x$ again. The Fourier transform $\widetilde{V}_{0}(k)=\widetilde{V}_{0}(-k)$ can be expressed in terms of the modified Bessel function $K_{0}(z)$ [46],

$$
\widetilde{V}_{0}(k)=\frac{4 e^{2}}{\kappa \pi} \int_{0}^{\pi / 2} d \varphi K_{0}\left(k \sqrt{a_{z}^{2}+4 R^{2} \sin ^{2} \varphi}\right) .
$$

Using asymptotic properties of $K_{0}(z)$, we then find the long-wavelength form valid for $k R \ll 1$,

$$
\widetilde{V}_{0}(k)=\frac{2 e^{2}}{\kappa}\left(|\ln k R|+c_{0}\right),
$$

where $c_{0}=-\gamma+(\pi / 2) \ln 2 \simeq 0.51$ with Euler's constant $\gamma$. The logarithmic singularity at small wavevectors is a direct consequence of the long-range tail of $V_{0}(x)$.

If one starts instead from an on-site Hubbard interaction 41, 42,

$$
U\left(\boldsymbol{r}-\boldsymbol{r}^{\prime}\right)=U \delta_{\sigma,-\sigma^{\prime}} \delta_{p p^{\prime}} \delta\left(\boldsymbol{r}-\boldsymbol{r}^{\prime}\right),
$$

one finds from Eq. (2.12) the 1D forward scattering potential

$$
V_{0}(x)=\frac{U}{2 \pi R} \delta_{\sigma,-\sigma^{\prime}} \delta\left(x-x^{\prime}\right) .
$$

The characteristic $1 / R$ scaling of the effective $1 \mathrm{D}$ interaction has also been found in Ref. 42.

For $\left|x-x^{\prime}\right| \gg a$, our continuum calculation leading to $\delta V_{p}\left(x-x^{\prime}\right)=0$ is justified. However, for $\left|x-x^{\prime}\right| \leq a$, one has to be more careful. Here an additional $\alpha \mathrm{FS}$ term beyond Eq. (2.15) arises due to the hard core of the Coulomb interaction. To study this term, we put $x=x^{\prime}$ and evaluate $\delta V_{p}(0)$ directly on the wrapped graphite network shown in Fig. 3. For simplicity, we again consider an armchair SWNT, albeit the same result [see Eq. (2.23) below] is found for other nanotube geometries. We now write

$$
\delta V_{p}\left(x-x^{\prime}\right) \simeq-2 f_{p} \delta\left(x-x^{\prime}\right),
$$

and since $f_{p}=f_{-p}=f$ [see below], we obtain the additional $\alpha \mathrm{FS}$ contribution in the form

$$
H_{\alpha \mathrm{FS}}^{(1)}=-f \int d x \sum_{p \alpha \alpha^{\prime} \sigma \sigma^{\prime}} \psi_{p \alpha \sigma}^{\dagger} \psi_{-p \alpha^{\prime} \sigma^{\prime}}^{\dagger} \psi_{-p \alpha^{\prime} \sigma^{\prime}} \psi_{p \alpha \sigma}
$$

The coupling constant $f>0$ depends on the nanotube geometry only and is evaluated for the $(n, n)$ case next.

To calculate $\delta V_{p}(0)$ on the wrapped graphite lattice, we start from the microscopic arrangement of carbon atoms around the waist of the armchair SWNT, see Fig. 3. On the length scale $\left|y-y^{\prime}\right| \leq 2 \pi R$, it is always justified to use the externally unscreened Coulomb interaction potential (2.9). Denoting the locations of carbon atoms on sublattice $p=+$ (the circles in Fig. 3) as $y_{k}=2 \pi(k / n) R=3 k d$ with $k=1, \ldots, n$, and discretizing Eq. (2.14), we obtain from Eq. (2.22)

$$
\begin{gathered}
2 f_{p} / a=(3 d / 2 \pi R)^{2} \sum_{l, k=1}^{n}\left\{U\left(0, y_{l}-y_{k}\right)\right. \\
\left.-U\left(0, y_{l}-y_{k}+p d\right)\right\} .
\end{gathered}
$$

The summation extends over the $p=+$ sites only, and inserting Eq. (2.9) yields with $R=\sqrt{3} n a / 2 \pi$,

$$
\begin{array}{r}
2 f_{p} / a=\frac{e^{2}}{2 n^{2} \kappa R} \sum_{l, k}\left\{\frac{1}{\sqrt{\sin ^{2}[(l-k) \pi / n]+\left(a_{z} / 2 R\right)^{2}}}\right. \\
\left.-\frac{1}{\sqrt{\sin ^{2}[(l-k+p / 3) \pi / n]+\left(a_{z} / 2 R\right)^{2}}}\right\} .
\end{array}
$$

The singular contributions are picked up from $l=k$ and $l=n-k$ and yield the $p$-independent contribution

$$
f / a=\gamma_{f} e^{2} / R,
$$

with the order-of-magnitude estimate

$$
\gamma_{f}=\frac{\sqrt{3} a}{2 \pi \kappa a_{z}}\left[1-\frac{1}{\sqrt{1+a^{2} / 3 a_{z}^{2}}}\right] \approx 0.05 .
$$

It can be checked easily that the remaining terms in the summation (2.24) are negligible against the singular contribution leading to Eq. 2.25). The prefactor $\gamma_{f}$ is independent of $n$ such that $f \sim 1 / n$. Parenthetically, in the language of the Hubbard-like models employed in Refs. 41, 42], we have $f / a=U-V$, where $U \approx e^{2} / R$ is the on-site and $V$ the nearest-neighbor Coulomb interaction. According to Eq. (2.25), this difference is small compared to $U$. Using a strictly on-site Hubbard model $(V=0)$ is therefore never realistic, even in the presence of close-by screening gates. 


\section{Backward scattering}

Let us now discuss the $\alpha \mathrm{BS}$ contributions depicted in Fig. 2. For backscattering processes, we have $\alpha_{1}=$ $-\alpha_{2}=\alpha_{3}=-\alpha_{4}=\alpha$ in Eq. (2.10), and from Eq. 2.11) the corresponding $1 \mathrm{D}$ interaction potential for $\alpha \mathrm{BS}$ processes reads

$$
V_{\alpha}^{p p^{\prime}}\left(x-x^{\prime}\right)=e^{2 i \alpha k_{F}\left(x-x^{\prime}\right)} V_{\alpha \mathrm{FS}}^{p p^{\prime}}\left(x-x^{\prime}\right) .
$$

Because of the rapidly oscillating phase factor, the only non-vanishing contribution to $H_{\alpha \text { BS }}$ comes from $\left|x-x^{\prime}\right| \leq$ $a$, i.e., we can effectively take a local interaction. According to Eq. (2.10), the $\alpha \mathrm{BS}$ contribution must then be of the general form

$H_{\alpha \mathrm{BS}}=\frac{1}{2} \int d x \sum_{p p^{\prime} \alpha \sigma \sigma^{\prime}}\left(b+p p^{\prime} b^{\prime}\right) \psi_{p \alpha \sigma}^{\dagger} \psi_{p^{\prime}-\alpha \sigma^{\prime}}^{\dagger} \psi_{p^{\prime} \alpha \sigma^{\prime}} \psi_{p-\alpha \sigma}$

The term corresponding to $b^{\prime}$ is irrelevant 447 and is omitted in what follows. The effective coupling constant $b>0$ is then determined by the Fourier transform of $V_{0}(x)$ at $k=2 k_{F}$,

$$
b=\widetilde{V}_{0}\left(2 k_{F}\right)
$$

and the backscattering contribution is

$$
H_{\alpha \mathrm{BS}}=\frac{b}{2} \int d x \sum_{p p^{\prime} \alpha \sigma \sigma^{\prime}} \psi_{p \alpha \sigma}^{\dagger} \psi_{p^{\prime}-\alpha \sigma^{\prime}}^{\dagger} \psi_{p^{\prime} \alpha \sigma^{\prime}} \psi_{p-\alpha \sigma}
$$

For the unscreened interaction (2.9), using $2 k_{F} R=$ $4 n / \sqrt{3} \gg 1$ and asymptotic properties of the Bessel function, we obtain from Eq. 2.18)

$$
b / a=\gamma_{b} e^{2} / R
$$

with the order-of-magnitude estimate

$$
\gamma_{b} \approx \frac{3}{2 \pi^{2} \kappa} \approx 0.1
$$

The prefactor $\gamma_{b}$ is independent of $n$, and thus $b \sim 1 / n$. This calculation for unscreened interactions predicts $b \approx$ $f$. If the Coulomb interaction is externally screened, however, a qualitatively different situation can arise. Now $f$ is still given by Eq. (2.25), but $b$ can become significantly larger, see Eq. (2.27). Therefore it is possible to have $b \gg f$ in the presence of screening gates.

\section{BOSONIZATION}

According to Sec. III, the low-energy theory of armchair SWNTs away from lattice commensurabilities is described by the Hamiltonian

$$
H=H_{0}+H_{\alpha \mathrm{FS}}^{(0)}+H_{\alpha \mathrm{FS}}^{(1)}+H_{\alpha \mathrm{BS}} .
$$

This model is equivalent to two spin- $\frac{1}{2}$ fermion chains coupled in a rather special way by the interactions, but without interchain single-particle hopping. As described below, it is in that respect that our theory differs from the standard two-chain problem of coupled Luttinger liquids investigated, e.g., in Refs. 48 [54]. The standard twochain model is usually derived by coupling two Hubbard chains via a transverse hopping matrix element [55], and has been used to study, e.g., the stability of Luttinger liquid behavior with respect to interchain coupling [56 58]. In contrast, the modified model (3.1) describes the properties of a SWNT. Our solution of this model proceeds in fact quite similar to the standard two-chain case. In particular, we now follow the bosonization route taken in Refs. 49 51] and especially in Ref. [52]. For a general review of bosonization, see Ref. 23.

In order to proceed, we need to bring the noninteracting Hamiltonian (2.6) into a standard form of the 1D Dirac model. This is accomplished by switching to right- and left-movers $(r= \pm)$ which are linear combinations of the sublattice states $p= \pm$. In particular, we have

$$
\psi_{p \alpha \sigma}(x)=\sum_{r} \widetilde{U}_{p r} \widetilde{\psi}_{r \alpha \sigma}(x)
$$

where the unitary operator $\widetilde{U}$ fulfills $\widetilde{U}^{\dagger} \sigma_{y} \widetilde{U}=\sigma_{z}$. Since the indices of $\widetilde{U}$ are $p= \pm$ and $r= \pm$, the $2 \times 2$ matrix representation $\widetilde{U}=(1 / \sqrt{2})[(1, i),(1,-i)]$ is easily found. The non-interacting Hamiltonian (2.6) then reads

$$
H_{0}=-i v \sum_{r \alpha \sigma} r \int d x \widetilde{\psi}_{r \alpha \sigma}^{\dagger} \partial_{x} \widetilde{\psi}_{r \alpha \sigma}
$$

Furthermore, the $1 \mathrm{D}$ density operator $\rho_{p \alpha \sigma}(x)$ entering Eq. 2.16) becomes

$$
\rho_{p \alpha \sigma}=\psi_{p \alpha \sigma}^{\dagger} \psi_{p \alpha \sigma}=\frac{1}{2} \sum_{r= \pm}\left(\widetilde{\rho}_{r \alpha \sigma}+p \widetilde{\psi}_{r \alpha \sigma}^{\dagger} \widetilde{\psi}_{-r \alpha \sigma}\right)
$$

where $\widetilde{\rho}_{r \alpha \sigma}(x)=\widetilde{\psi}_{r \alpha \sigma}^{\dagger}(x) \widetilde{\psi}_{r \alpha \sigma}(x)$. The 1D density defined in Eq. (2.16) is therefore equivalently expressed as

$$
\rho(x)=\sum_{r \alpha \sigma} \widetilde{\psi}_{r \alpha \sigma}^{\dagger}(x) \widetilde{\psi}_{r \alpha \sigma}(x) .
$$

In this representation, it is convenient to apply the bosonization formula [23],

$$
\widetilde{\psi}_{r \alpha \sigma}(x)=\frac{\eta_{r \alpha \sigma}}{\sqrt{2 \pi a}} \exp \left\{i q_{F} r x+i k_{F} \alpha x+i \varphi_{r \alpha \sigma}\right\}
$$

For simplicity, we have incorporated the spatial dependence due to the Bloch functions (2.2) into the 1D 
fermion operator. The chiral bosonic phase fields obey the commutator algebra

$$
\left[\varphi_{r \alpha \sigma}(x), \varphi_{r^{\prime} \alpha^{\prime} \sigma^{\prime}}\left(x^{\prime}\right)\right]_{-}=-i \pi r \delta_{r r^{\prime}} \delta_{\alpha \alpha^{\prime}} \delta_{\sigma \sigma^{\prime}} \operatorname{sgn}\left(x-x^{\prime}\right) .
$$

From these relations, the fermion operator $\widetilde{\psi}_{r \alpha \sigma}(x)$ has indeed the correct anticommutator algebra on a given branch $(r \alpha \sigma)$. To enforce anticommutator relations between different branches, we need the Majorana fermions (Klein factors) $\eta_{r \alpha \sigma}=\eta_{r \alpha \sigma}^{\dagger}$. They obey

$$
\left[\eta_{r \alpha \sigma}, \eta_{r^{\prime} \alpha^{\prime} \sigma^{\prime}}\right]_{+}=2 \delta_{r r^{\prime}} \delta_{\alpha \alpha^{\prime}} \delta_{\sigma \sigma^{\prime}} .
$$

We note that the usual zero modes [23] have to be incorporated in the fields $\varphi_{r \alpha \sigma}$ as they are not contained in the Majorana fermions. However, they matter only if one is interested in finite-size effects. Finally, from Eq. (3.6), the density $\widetilde{\rho}_{r \alpha \sigma}$ now takes the form

$$
\tilde{\rho}_{r \alpha \sigma}(x)=\frac{q_{F}}{2 \pi}+\frac{r}{2 \pi} \partial_{x} \varphi_{r \alpha \sigma}(x) .
$$

The wave vector $q_{F}$, which must be carefully distinguished from the Fermi vector $k_{F}$, is related to the band filling. By varying a suitable gate voltage [18], one can easily adjust the Fermi energy and hence the band filling (see Fig. 11). Relative to the unbiased half-filled case $E_{F}=0$, an average excess density $\delta \rho$ gives rise to a non-zero $q_{F}=\pi \delta \rho / 4$ and hence $E_{F}=v q_{F}$. We consider $\left|q_{F}\right| \ll k_{F}$ in the following, since otherwise the low-energy continuum approximation underlying our approach might break down.

At this stage, it is natural to introduce the standard linear combinations $\theta_{j \delta}(x)$ and their dual fields $\phi_{j \delta}(x)$ subject to the algebra

$$
\left[\phi_{j \delta}(x), \theta_{j^{\prime} \delta^{\prime}}\left(x^{\prime}\right)\right]=-(i / 2) \delta_{j j^{\prime}} \delta_{\delta \delta^{\prime}} \operatorname{sgn}\left(x-x^{\prime}\right) .
$$

The bosonic phase fields $\theta_{j \delta}(x)$ for the total and relative $(\delta= \pm)$ charge $(j=c)$ and spin $(j=s)$ channels are constructed as

$$
\begin{aligned}
& \theta_{c+}=\frac{1}{4 \sqrt{\pi}} \sum_{r \alpha \sigma} r \varphi_{r \alpha \sigma}, \\
& \theta_{c-}=\frac{1}{4 \sqrt{\pi}} \sum_{r \alpha \sigma} r \alpha \varphi_{r \alpha \sigma}, \\
& \theta_{s+}=\frac{1}{4 \sqrt{\pi}} \sum_{r \alpha \sigma} r \sigma \varphi_{r \alpha \sigma}, \\
& \theta_{s-}=\frac{1}{4 \sqrt{\pi}} \sum_{r \alpha \sigma} r \alpha \sigma \varphi_{r \alpha \sigma} .
\end{aligned}
$$

Their dual fields $\phi_{j \delta}$ are defined similarly by omitting the $r$ factor in these relations. The back-transformation reads

$$
\begin{aligned}
\varphi_{r \alpha \sigma} & =\frac{\sqrt{\pi}}{2}\left\{\phi_{c+}+r \theta_{c+}+\alpha \phi_{c-}+r \alpha \theta_{c-}\right. \\
& \left.+\sigma \phi_{s+}+r \sigma \theta_{s+}+\alpha \sigma \phi_{s-}+r \alpha \sigma \theta_{s-}\right\},
\end{aligned}
$$

such that Eq. (3.7) is recovered from Eq. (3.9). The total electron density relative to the half-filled situation reads from Eq. (3.5)

$$
\rho(x)=4 q_{F} / \pi+\frac{2}{\sqrt{\pi}} \partial_{x} \theta_{c+}(x),
$$

and the continuity equation then yields the current,

$$
I=\frac{2 e}{\sqrt{\pi}} \partial_{t} \theta_{c+}(x, t),
$$

which can be evaluated, say, at $x=0$.

In order to arrive at the bosonized form of the model (3.1), we need to specify the Majorana fermions $\eta_{r \alpha \sigma}$ in Eq. (3.6). Since the Hamiltonian contains only the spinconserving products

$$
A_{ \pm \pm}=\eta_{r \alpha \sigma} \eta_{ \pm r \pm \alpha \sigma}
$$

these can be represented using standard Pauli matrices 24, 59. Besides $A_{++}=1$, we choose

$$
A_{+-}=i \alpha \sigma_{x}, A_{-+}=i r \alpha \sigma_{z}, A_{--}=-i r \sigma_{y} .
$$

To show that Eq. (3.14) leads to a valid representation for the Klein factors, we have to check all possible products of $A_{ \pm \pm}$with each other. For instance, the product

$$
A_{+-} A_{-+}=\eta_{r \alpha \sigma} \eta_{r-\alpha \sigma} \eta_{r \alpha \sigma} \eta_{-r \alpha \sigma}
$$

gives $\left(i \alpha \sigma_{x}\right)\left(i r \alpha \sigma_{z}\right)=i r \sigma_{y}$ according to Eq. (3.14). On the other hand, using the anticommutator relation (3.8) gives for Eq. (3.15) $-\eta_{r-\alpha \sigma} \eta_{-r \alpha \sigma}$, which is again $i r \sigma_{y}$ from Eq. (3.14). Similarly, all other products are consistent with the algebra (3.8), and one can indeed use the representation (3.13) with Eq. (3.14). Of course, our choice (3.14) is not unique, and one can find other possibilities. However, final expressions for physically observable quantities do not depend on this choice.

The bosonized expressions for the various terms in Eq. (3.1) read 60]

$$
\begin{aligned}
H_{0} & =\sum_{j \delta} \frac{v_{j \delta}}{2} \int d x\left[K_{j \delta}\left(\partial_{x} \phi_{j \delta}\right)^{2}+K_{j \delta}^{-1}\left(\partial_{x} \theta_{j \delta}\right)^{2}\right] \\
H_{\alpha \mathrm{FS}}^{(0)} & =\frac{2}{\pi} \int d x d x^{\prime} \partial_{x} \theta_{c+}(x) V_{0}\left(x-x^{\prime}\right) \partial_{x^{\prime}} \theta_{c+}\left(x^{\prime}\right) \\
H_{\alpha \mathrm{FS}}^{(1)} & =\frac{f}{(\pi a)^{2}} \int d x\left[-\cos \left(\sqrt{4 \pi} \theta_{c-}\right) \cos \left(\sqrt{4 \pi} \theta_{s-}\right)\right. \\
& -\cos \left(\sqrt{4 \pi} \theta_{c-}\right) \cos \left(\sqrt{4 \pi} \theta_{s+}\right) \\
& \left.+\cos \left(\sqrt{4 \pi} \theta_{s+}\right) \cos \left(\sqrt{4 \pi} \theta_{s-}\right)\right] \\
H_{\alpha \mathrm{BS}} & =\frac{b}{(\pi a)^{2}} \int d x\left[\cos \left(\sqrt{4 \pi} \theta_{c-}\right) \cos \left(\sqrt{4 \pi} \theta_{s-}\right)\right. \\
& +\cos \left(\sqrt{4 \pi} \theta_{c-}\right) \cos \left(\sqrt{4 \pi} \phi_{s-}\right) \\
& \left.+\cos \left(\sqrt{4 \pi} \theta_{s-}\right) \cos \left(\sqrt{4 \pi} \phi_{s-}\right)\right] .
\end{aligned}
$$


The correct signs for the various terms in Eqs. (3.18) and (3.19) are crucial and necessitate a correct choice for the Majorana fermion products, see Eq. (3.14). Although bosonization of Eq. (3.3) gives $K_{j \delta}=1$ in Eq. (3.16), interactions renormalize these parameters. In particular, in the long-wavelength limit, $H_{\alpha \mathrm{FS}}^{(0)}$ can be incorporated into $H_{0}$ by putting

$$
K_{c+}=K=\left\{1+4 \widetilde{V}_{0}(k \simeq 0) / \pi v\right\}^{-1 / 2} \leq 1
$$

while for all other channels $[(j \delta) \neq(c+)]$, the coupling constant $f$ gives rise to the renormalization

$$
K_{j \delta}=1+f / \pi v \geq 1 .
$$

The corresponding renormalization of $K_{c+}$ due to $f \neq 0$ can be neglected against the large effect of Eq. (3.17). The velocities of the different modes in Eq. (3.16) are then given by

$$
v_{j \delta}=v / K_{j \delta}
$$

Clearly, the charged $(c+)$ mode propagates with significantly higher velocity than the three neutral modes. There is a further renormalization of the values given by Eq. (3.22) due to the $\alpha \mathrm{BS}$ contribution. However, this effect is very small and does not affect the power laws discussed below. We shall therefore neglect velocity renormalizations beyond Eq. (3.22) in the following.

For the long-ranged interaction (2.19), the logarithmic singularity in Eq. (3.20 has a natural infrared cutoff at $k=2 \pi / L$ due to the finite length of the nanotube. For temperatures $k_{B} T \gg \hbar v / L$, thermal effects provide a higher cut-off. Considering low temperatures, $k_{B} T \ll$ $\hbar v / L$, we then obtain

$$
K=\left\{1+\frac{8 e^{2}}{\pi \kappa \hbar v}[\ln (L / 2 \pi R)+0.51]\right\}^{-1 / 2}
$$

which predicts $K \rightarrow 0$ for $L \rightarrow \infty$. Since $\hbar c / e^{2} \simeq 137$, we get with $v=8 \times 10^{5} \mathrm{~m} / \mathrm{sec}$ the estimate $e^{2} / \hbar v=$ $\left(e^{2} / \hbar c\right)(c / v) \approx 2.7$, and therefore $K \simeq 0.18$ for the $L=$ $3 \mu \mathrm{m}$ tube of Ref. [18]. Quite generally, the parameter $K$ gives the appropriate measure of the correlation strength 22, 23. The rather small value of $K$ found here implies that a single-wall armchair nanotube is a very strongly correlated system, which should display pronounced nonFermi liquid effects.

\section{RENORMALIZATION GROUP ANALYSIS}

The Hamiltonian given by Eqs. 3.16)-(3.19) does not allow for an exact solution. To proceed, we investigate the nonlinear terms associated with the coupling constants $f$ and $b$ by using the perturbative RG method
[61. Since there is no renormalization in the charged $(c+)$ sector, in the following $(j \delta)$ includes only the combinations $(c-),(s+)$, and $(s-)$. There are eight scaling operators perturbing the critical $b=f=0$ model that need to be taken into account. They are given by

$$
\begin{aligned}
V_{1} & =\frac{1}{\pi} \cos \left(\sqrt{4 \pi} \theta_{c-}\right) \cos \left(\sqrt{4 \pi} \theta_{s-}\right), \\
V_{2} & =\frac{1}{\pi} \cos \left(\sqrt{4 \pi} \theta_{c-}\right) \cos \left(\sqrt{4 \pi} \theta_{s+}\right), \\
V_{3} & =\frac{1}{\pi} \cos \left(\sqrt{4 \pi} \theta_{s-}\right) \cos \left(\sqrt{4 \pi} \theta_{s+}\right), \\
V_{4} & =\frac{1}{\pi} \cos \left(\sqrt{4 \pi} \theta_{c-}\right) \cos \left(\sqrt{4 \pi} \phi_{s-}\right), \\
V_{5} & =\frac{1}{\pi} \cos \left(\sqrt{4 \pi} \theta_{s+}\right) \cos \left(\sqrt{4 \pi} \phi_{s-}\right), \\
V_{j \delta} & =\frac{1}{2}\left[-\left(\partial_{x} \phi_{j \delta}\right)^{2}+\left(\partial_{x} \theta_{j \delta}\right)^{2}\right]
\end{aligned}
$$

with associated scaling fields (coupling constants) $g_{i}(\ell)$ (where $i=1, \ldots, 5)$ and $g_{j \delta}(\ell)$. Here $d \ell=-d \ln \omega_{c}$ denotes the standard RG flow parameter, i.e., one decreases the high-energy bandwidth cutoff $\omega_{c}$ and compensates this decrease by adjusting the coupling constants. Within the reach of the perturbative $\mathrm{RG}$, the coupling constants $g_{j \delta}$ are related to the $K_{j \delta}$ parameters by $K_{j \delta}=1-2 g_{j \delta}$. The initial values of the coupling constants are

$$
\begin{aligned}
& g_{1}(0)=(b-f) / \pi v \\
& g_{2}(0)=-g_{3}(0)=2 g_{j \delta}(0)=-f / \pi v, \\
& g_{4}(0)=b / \pi v \\
& g_{5}(0)=0
\end{aligned}
$$

The operator $V_{5}$ is not present in the original Hamiltonian but will be generated during the renormalization process. Furthermore, we have omitted the operator

$$
V^{\prime}=\frac{1}{\pi} \cos \left(\sqrt{4 \pi} \theta_{s_{-}}\right) \cos \left(\sqrt{4 \pi} \phi_{s_{-}}\right),
$$

which is present in Eq. (3.19). This operator stays exactly marginal in all orders and thus decouples completely from the problem. In fact, by using the Majorana refermionization procedure in Sec. $\mathrm{V}$, this term is seen to vanish.

It is straightforward to derive the second-order $\mathrm{RG}$ equations, e.g., by using poor man's scaling or, more elegantly, the operator product expansion technique [61]. The resulting equations read

$$
\begin{aligned}
& \frac{d g_{1}}{d \ell}=-g_{2} g_{3}+g_{1}\left(g_{c-}+g_{s-}\right) \\
& \frac{d g_{2}}{d \ell}=-g_{1} g_{3}-g_{4} g_{5}+g_{2}\left(g_{c-}+g_{s+}\right) \\
& \frac{d g_{3}}{d \ell}=-g_{1} g_{2}+g_{3}\left(g_{s+}+g_{s-}\right) \\
& \frac{d g_{4}}{d \ell}=-g_{2} g_{5}+g_{4}\left(g_{c-}-g_{s-}\right)
\end{aligned}
$$




$$
\begin{aligned}
\frac{d g_{5}}{d \ell} & =-g_{2} g_{4}+g_{5}\left(g_{s+}-g_{s-}\right), \\
\frac{d g_{c-}}{d \ell} & =\frac{1}{2}\left(g_{1}^{2}+g_{2}^{2}+g_{4}^{2}\right), \\
\frac{d g_{s+}}{d \ell} & =\frac{1}{2}\left(g_{2}^{2}+g_{3}^{2}+g_{5}^{2}\right), \\
\frac{d g_{s-}}{d \ell} & =\frac{1}{2}\left(g_{1}^{2}+g_{3}^{2}-g_{4}^{2}-g_{5}^{2}\right) .
\end{aligned}
$$

We shall first study these equations in two limiting cases, namely either $b=0$ or $f=0$.

Let us start with the case $b=0$, where the coupling constants $g_{4}$ and $g_{5}$ stay identically zero. Putting

$$
G=-g_{1}=-g_{2}=g_{3}
$$

with the initial condition $G(0)=f / \pi v>0$, and

$$
\bar{G}=2 g_{c-}=2 g_{s-}=2 g_{s+}
$$

with $\bar{G}(0)=-f / \pi v<0$, the RG equations read

$$
\frac{d G}{d \ell}=-G^{2}+\bar{G} G, \quad \frac{d \bar{G}}{d \ell}=2 G^{2} .
$$

This permits the solution $G=-\bar{G}$ obeying $d G / d \ell=$ $-2 G^{2}$. Since $G(0)>0$, all coupling constants flow to zero as $\ell \rightarrow \infty$,

$$
G(\ell)=\frac{G(0)}{1+2 G(0) \ell} .
$$

Therefore, for $b=0$, the contribution $H_{\alpha \mathrm{FS}}^{(1)}$ in Eq. 3.18) is marginally irrelevant.

Next we turn to the case $f=0$, where one can immediately put $g_{2}=g_{3}=g_{5}=g_{s+}=0$. Furthermore, we are free to choose the isotropic solution

$$
g=g_{1}=g_{4},
$$

with the initial condition $g(0)=b / \pi v>0$. Then one can also put $g_{s-}=0$ and is left with the Kosterlitz-Thouless equations

$$
\frac{d g}{d \ell}=g g_{c-}, \quad \frac{d g_{c-}}{d \ell}=g^{2} .
$$

Therefore both $g$ and $g_{c-}$ will flow to strong coupling, and the backscattering part $H_{\alpha \mathrm{BS}}$ is marginally relevant. The physical picture emerging for the special case $f=0$ is elucidated in Sec. V. From this analysis and the numerical solution of the general RG equations (4.7)-(4.14) for $f \neq 0$, it is apparent that $b$ is always a marginally relevant coupling constant.

The next question to be addressed is whether the initially irrelevant $H_{\alpha \mathrm{FS}}^{(1)}$ becomes relevant near the emerging strong coupling fixed point. To study this issue, let us assume $b \gg f$ and linearize the RG equations in the initially small coupling constants $g_{2}, g_{3}, g_{5}$, and $g_{j}$. Then $g_{s+}$ decouples, and we introduce the new coupling constants

$$
g=\left(g_{1}+g_{4}\right) / 2, \quad \bar{g}=g_{1}-g_{4},
$$

with initial values $g(0)=b / \pi v$ and $\bar{g}(0)=-f / \pi v$. Thereby we recover the Kosterlitz-Thouless equations (4.15), so that the above strong-coupling behavior of $b$ is basically unaltered, i.e., the coupling constants $g$ and $g_{c-}$ flow to strong coupling, $g \rightarrow g^{*}>0$ and $K_{c-} \rightarrow K_{c-}^{*}<1$. The remaining $\mathrm{RG}$ equations read

$$
\begin{aligned}
\frac{d \bar{g}}{d \ell} & =2 g g_{s-}, \\
\frac{d g_{2}}{d \ell} & =-g\left(g_{3}+g_{5}\right), \\
\frac{d g_{3}}{d \ell} & =\frac{d g_{5}}{d \ell}=-g g_{2}, \\
\frac{d g_{s-}}{d \ell} & =\bar{g} g .
\end{aligned}
$$

Therefore, $\bar{g}(\ell)$ also reaches the strong coupling regime, $\bar{g} \rightarrow \bar{g}^{*}<0$, and similarly, $K_{s-} \rightarrow K_{s-}^{*}>1$. Furthermore, it follows readily that $g_{2}, g_{3}$ and $g_{5}$ flow to strong coupling (with $g_{3}-g_{5}$ staying constant). We conclude that terms associated with the coupling constant $f$ will become relevant near the strong-coupling point $b \rightarrow b^{*}$, which therefore represents only an intermediate fixed point.

This conclusion remains valid if the assumption $b \gg f$ is relaxed. In Fig. 1, the numerical integration of the RG flow equations (4.7)-(4.14) is shown for realistic values of the coupling constants $b$ and $f$. Clearly, all coupling constants go to the strong-coupling regime, albeit $g_{4}$ associated with the backscattering contribution is dominant. Only at sufficiently low energy scales, i.e., for large values of the flow parameter $\ell$, the nonlinear forward scattering term $H_{\alpha \mathrm{FS}}^{(1)}$ becomes important. Finally, in Fig. 5 we show the RG flow for different initial conditions corresponding to $f>b$. The coupling constants $g_{2}$ and $g_{3}$ associated with the forward scattering contribution are initially irrelevant. However, they eventually become relevant and flow to strong coupling as $\ell \rightarrow \infty$. In the end, all coupling constants flow into the strong-coupling regime again.

\section{MAJORANA REFERMIONIZATION}

We start this section by presenting a solution for the special case $f=0$. This solution proceeds by Majorana refermionization and is similar to the one discussed by Schulz in the context of the standard two-chain problem [52]. For $f=0$, the nonlinearity affects only the relative $(\delta=-)$ channels, for which the bosonized Hamiltonian reads from Eqs. (3.16) and (3.19) 


$$
\begin{aligned}
H\left(c-, s_{-}\right) & =\frac{v}{2} \int d x \sum_{j=c, s}\left[\left(\partial_{x} \phi_{j-}\right)^{2}+\left(\partial_{x} \theta_{j-}\right)^{2}\right] \\
& +\frac{b}{(\pi a)^{2}} \int d x\left[\cos \left(\sqrt{4 \pi} \theta_{c-}\right) \cos \left(\sqrt{4 \pi} \theta_{s-}\right)\right. \\
& +\cos \left(\sqrt{4 \pi} \theta_{c-}\right) \cos \left(\sqrt{4 \pi} \phi_{s-}\right) \\
& \left.+\cos \left(\sqrt{4 \pi} \theta_{s-}\right) \cos \left(\sqrt{4 \pi} \phi_{s-}\right)\right] .
\end{aligned}
$$

Let us define new effective fermion operators for the relative charge and spin $(j=c, s)$ channels. Their right- and left-moving components $(p= \pm=R, L)$ can be written in terms of the bosonic phase fields,

$$
\psi_{j p}=\frac{\eta_{j p}}{\sqrt{2 \pi a}} \exp \left\{-i \sqrt{\pi}\left(p \theta_{j-}+\phi_{j-}\right)\right\} .
$$

Then we have

$$
\begin{aligned}
& \cos \left[\sqrt{4 \pi} \theta_{j-}\right]=-\eta_{j R} \eta_{j L} \pi a\left(\psi_{j R}^{\dagger} \psi_{j L}-\psi_{j L}^{\dagger} \psi_{j R}\right) \\
& \cos \left[\sqrt{4 \pi} \phi_{j-}\right]=-\eta_{j R} \eta_{j L} \pi a\left(\psi_{j R}^{\dagger} \psi_{j L}^{\dagger}-\psi_{j L} \psi_{j R}\right)
\end{aligned}
$$

Next we express these two (complex) Dirac fermions in terms of four (real) Majorana fermions $\xi_{j p}(x)$. Here $j=$ 1,2 corresponds to the $(s-)$ channel, and $j=3,4$ to $(c-)$,

$$
\begin{aligned}
& \psi_{s, R / L}=\frac{1}{\sqrt{2}}\left(\xi_{1, R / L}(x)+i \xi_{2, R / L}(x)\right), \\
& \psi_{c, R / L}=\frac{1}{\sqrt{2}}\left(\xi_{3, R / L}(x)+i \xi_{4, R / L}(x)\right) .
\end{aligned}
$$

The Majorana fermion operators obey the algebra

$$
\left[\xi_{j p}(x), \xi_{j^{\prime} p^{\prime}}\left(x^{\prime}\right)\right]_{+}=\delta_{j j^{\prime}} \delta_{p p^{\prime}} \delta\left(x-x^{\prime}\right),
$$

and by using Eq. (5.2) we find

$$
\begin{aligned}
\cos \left[\sqrt{4 \pi} \theta_{s-}\right] & =-i \pi a\left(\xi_{1 R} \xi_{1 L}+\xi_{2 R} \xi_{2 L}\right), \\
\cos \left[\sqrt{4 \pi} \phi_{s-}\right] & =-i \pi a\left(\xi_{1 R} \xi_{1 L}-\xi_{2 R} \xi_{2 L}\right), \\
\cos \left[\sqrt{4 \pi} \theta_{c-}\right] & =i \pi a\left(\xi_{3 R} \xi_{3 L}+\xi_{4 R} \xi_{4 L}\right),
\end{aligned}
$$

where the Klein factors have been chosen as $\eta_{c R} \eta_{c L}=$ $-\eta_{s R} \eta_{s L}=-i$. This choice is dictated by the condition that the interaction term is marginally relevant, see below.

As already mentioned, one can see from this representation that the operator

$$
\cos \left[\sqrt{4 \pi} \theta_{s-}\right] \cos \left[\sqrt{4 \pi} \phi_{s-}\right]
$$

can effectively be put to zero. To be precise, upon point splitting [23,61], it yields highly irrelevant operators involving spatial derivatives of the Majorana fields. The corresponding term in Eq. (5.1) can thus be omitted. Refermionization then yields [we put $v=1$ in the intermediate steps]

$$
\begin{aligned}
H(c-, s-) & =-\frac{i}{2} \sum_{j=1}^{4} \int d x\left(\xi_{j R} \partial_{x} \xi_{j R}-\xi_{j L} \partial_{x} \xi_{j L}\right) \\
& +2 b \int d x\left(\xi_{3 R} \xi_{3 L}+\xi_{4 R} \xi_{4 L}\right) \xi_{1 R} \xi_{1 L} .
\end{aligned}
$$

In order to better understand the above model, it is instructive to rewrite it in terms of the following current operators,

$$
\begin{aligned}
I_{R}^{x}(x) & =i \xi_{1 R}(x) \xi_{3 R}(x), \\
I_{R}^{y}(x) & =-i \xi_{1 R}(x) \xi_{4 R}(x), \\
I_{R}^{z}(x) & =i \xi_{3 R}(x) \xi_{4 R}(x),
\end{aligned}
$$

and analogously for the left-movers. These currents obey the $S U(2)$ level-2 Kac-Moody algebra [23],

$$
\left[I^{a}(x), I^{b}(y)\right]_{-}=i \epsilon^{a b c} \delta(x-y) I^{c}(x)+\frac{i \delta_{a b}}{2 \pi} \delta^{\prime}(x-y) .
$$

In terms of the operators (5.6), the interacting part of the Hamiltonian (5.5) takes the form

$$
\int d x\left\{g_{\perp}\left[I_{R}^{x}(x) I_{L}^{x}(x)+I_{R}^{y}(x) I_{L}^{y}(x)\right]+g_{z} I_{R}^{z}(x) I_{L}^{z}(x)\right\} .
$$

The transverse coupling is $g_{\perp}=2 b$, while the longitudinal coupling $g_{z}$ is initially absent but will be generated under the renormalization. We thus can identify the model (5.5) as the $S U_{2}(2)$ anisotropic Wess-ZuminoWitten model, see, e.g., Ref. 23]. From the structure of the commutation relations, it immediately follows that the coupling constants $\left(g_{\perp}, g_{z}\right)$ obey the KosterlitzThouless equations

$$
\frac{d g_{\perp}}{d \ell}=\frac{1}{2 \pi} g_{\perp} g_{z}, \quad \frac{d g_{z}}{d \ell}=\frac{1}{2 \pi} g_{\perp}^{2} .
$$

Using the operator identity

$$
\begin{aligned}
\left\{\frac{1}{\pi a} \cos [\sqrt{4 \pi} \theta(x)]\right\}^{2} & =\frac{1}{(\pi a)^{2}} \cos [\sqrt{16 \pi} \theta(x)] \\
& -\frac{1}{\pi}\left(\partial_{x} \theta\right)^{2}+\text { const. }
\end{aligned}
$$

we conclude that the longitudinal coupling corresponds to a renormalization of $K_{c-}$, with $g_{z}=2 \pi g_{c-}$. Using this identification we recover the flow equations 4.15) and highlight the underlying reason for their KosterlitzThouless structure.

Of course, for $b>0$, the model flows to strong coupling, rendering the Majorana fields $\xi_{1}, \xi_{3}$ and $\xi_{4}$ massive. Notice, however, that the Majorana fermion $\xi_{2}$ stays massless, so that the $(s-)$ sector carries one massive $(j=1)$ and one massless branch. This behavior is due to the symmetric appearance of the dual fields $\theta_{s-}$ and $\phi_{s-}$ (self-duality) in Eq. (5.1). By virtue of the Heisenberg 
uncertainty relation, it is impossible to completely pin a self-dual field. However, the $(c-)$ sector is fully massive.

The masses of the massive branches can now safely be calculated by Majorana mean-field theory. We mention in passing that a standard self-consistent harmonic treatment 23] does not apply in this case since we have to deal with a marginally relevant perturbation. In the meanfield approximation, the interaction term in Eq. (5.5) is written as

$$
-2 i b \int d x\left(c_{1}\left[\xi_{3 R} \xi_{3 L}+\xi_{4 R} \xi_{4 L}\right]+2 c_{3} \xi_{1 R} \xi_{1 L}\right)
$$

with the ground-state averages

$$
\begin{aligned}
& c_{1}=i\left\langle\xi_{1 R} \xi_{1 L}\right\rangle \\
& c_{3}=c_{4}=i\left\langle\xi_{3 R} \xi_{3 L}\right\rangle=i\left\langle\xi_{4 R} \xi_{4 L}\right\rangle .
\end{aligned}
$$

At this point it is straightforward to diagonalize the mean-field Hamiltonian. This yields the self-consistency relations

$$
\begin{aligned}
& c_{1}=\frac{2 b c_{3}}{\pi} \ln \left(\omega_{c} / 4 b c_{3}\right), \\
& c_{3}=\frac{b c_{1}}{\pi} \ln \left(\omega_{c} / 2 b c_{1}\right),
\end{aligned}
$$

where $\omega_{c}=7.4 \mathrm{eV}$ is the bandwidth of the $\pi$ electrons [3]. The solution to these relations is $c_{3} \simeq c_{1} / \sqrt{2}$ with

$$
c_{1} \simeq \frac{\omega_{c}}{2 b} \exp [-\pi / \sqrt{2} b] .
$$

Comparing Eq. (5.10 with the usual mass term $-i m \int d x \xi_{R} \xi_{L}$ yields the masses $m_{j}$ of the massive Majorana fermion branches $j=1,3,4$. Apart from a factor $\sqrt{2}$, these masses are all equal and given by

$$
m_{b}=\omega_{c} \exp [-\pi v / \sqrt{2} b] .
$$

Since the coupling constant $b$ appears in the exponent of an exponentially small quantity, it is quite difficult to estimate the value of $m_{b}$. Taking the value (2.29) for $b$ yields an order-of-magnitude estimate for the associated temperature scale, $T_{b} \approx 0.1 \mathrm{mK}$. For well-screened interactions, however, $T_{b}$ can be orders of magnitude larger.

To proceed, since the $(c-)$ sector is fully massive, we now effectively put $\theta_{c-}=0$ in Eq. (5.1). Other degenerate solutions give the same physical results. Then we are confronted with the interaction Hamiltonian

$$
\frac{m_{b}}{2 \pi a} \int d x\left(\cos \left[\sqrt{4 \pi} \theta_{s-}\right]+\cos \left[\sqrt{4 \pi} \phi_{s-}\right]\right) .
$$

Self-duality implies that $\sin \left[\sqrt{\pi} \theta_{s_{-}}\right]$and $\sin \left[\sqrt{\pi} \phi_{s_{-}}\right]$must have the same scaling dimension. Similarly, the scaling dimensions of $\cos \left[\sqrt{\pi} \theta_{s_{-}}\right]$and $\cos \left[\sqrt{\pi} \phi_{s-}\right]$ must coincide. Although the fields $\theta_{s-}$ and $\phi_{s-}$ are not pinned, they still have a tendency to be pinned. Since $m_{b}>0$, this implies that the values of $\sin \left[\sqrt{\pi} \theta_{s_{-}}\right]$and $\sin \left[\sqrt{\pi} \phi_{s_{-}}\right]$are close to
1, while the corresponding cos-operators approach zero. Therefore the sin-operators will be correlated stronger than the cos-operators.

These simple arguments can be made quantitative as follows. The $(s-)$ sector is described by two decoupled Hamiltonians, $H=H_{1}\left[\xi_{1}\right]+H_{2}\left[\xi_{2}\right]$, for the Majorana fermions $\xi_{1}$ and $\xi_{2}$,

$$
\begin{aligned}
H_{j}\left[\xi_{j}\right] & =-\frac{i}{2} \int d x\left(\xi_{j R} \partial_{x} \xi_{j R}-\xi_{j L} \partial_{x} \xi_{j L}\right) \\
& -i m_{j} \int d x \xi_{j R} \xi_{j L},
\end{aligned}
$$

where the masses are given by $m_{1}=m_{b}$ and $m_{2}=0$. We now exploit the well-known correspondence between the 2D classical Ising model and 1D Majorana fermions 23, 62. The fermion mass $m_{j}$ sets the relevant energy scale, where the correlation length of the related Ising model should be proportional to $m_{j}$. Therefore the Ising model related to $\xi_{2}$ will be critical $\left(T=T_{c}\right)$, while the Ising model for $\xi_{1}$ is above criticality (since $m_{1}>0$, we have $T>T_{c}$ ). Denoting the standard order operators for the two Ising models as $\sigma_{1,2}$, and disorder operators as $\mu_{1,2}$, the fusion rules [23,62] yield the correspondence

$$
\begin{aligned}
\cos \left[\sqrt{\pi} \theta_{s-}\right] & =\sigma_{1} \mu_{2}, \\
\cos \left[\sqrt{\pi} \phi_{s-}\right] & =\sigma_{1} \sigma_{2}, \\
\sin \left[\sqrt{\pi} \theta_{s-}\right] & =\mu_{1} \sigma_{2}, \\
\sin \left[\sqrt{\pi} \phi_{s-}\right] & =\mu_{1} \mu_{2} .
\end{aligned}
$$

These fusion rules state that for two given Ising models, products of the order/disorder operators determine the Majorana field operators, which in turn are composed out of the exponentials $\exp \left[ \pm i \sqrt{\pi} \theta_{s-}\right]$ and $\exp \left[ \pm i \sqrt{\pi} \phi_{s-}\right]$. The relation (5.13) is a valid representation of the fusion rules, see Ref. [23].

Since the first Ising model is above criticality, the average of the order operator is zero, $\left\langle\sigma_{1}\right\rangle=0$. However, the disorder operator $\mu_{1}$ has a finite average value, and correlation functions of $\cos \left[\sqrt{\pi} \theta_{s-}\right]$ and $\cos \left[\sqrt{\pi} \phi_{s-}\right]$ decay exponentially. From the exact solution of the 2D Ising model 62 one then immediately obtains

$$
\left\langle\sin \left[\sqrt{\pi} \theta_{s-}(x)\right] \sin \left[\sqrt{\pi} \theta_{s-}\left(x^{\prime}\right)\right]\right\rangle \sim\left|x-x^{\prime}\right|^{-1 / 4},
$$

with the same result for the $\sin \left[\sqrt{\pi} \phi_{s-}\right]$ correlator. The sin-operators therefore contain the Ising operator $\mu_{1}$ from the off-critical model which has a finite average. The other Ising operator then comes from a critical model $\left(m_{2}=0\right)$ and leads to the scaling dimension $\eta=1 / 8$, see Eq. (5.14). In contrast, the cos-operators contain the Ising operator $\sigma_{1}$ with zero expectation value. Note that for $b=0$ all these operators have scaling dimension $\eta=1 / 4$. The "halved" scaling dimension $\eta=1 / 8$ found for $b>0$ can be traced to the fact that one Majorana fermion becomes massive while the other stays massless. 
In the $(c-)$ sector, correlations of $\cos \left[\sqrt{\pi} \theta_{c-}\right]$ show longrange order while all other operators lead to exponential decay.

The results obtained so far by Majorana refermionization apply only to the intermediate fixed point characterized by $f=0$. As discussed in Sec. IV, while $H_{\alpha \mathrm{FS}}^{(1)}$ is irrelevant around the non-interacting fixed point, it becomes relevant near this intermediate strongcoupling point. The term $\sim \cos \left(\sqrt{4 \pi} \theta_{s+}\right) \cos \left(\sqrt{4 \pi} \theta_{s-}\right)$ in Eq. (3.18) stays marginal, but the two other terms become relevant with scaling dimension $\eta=1$. This renders the $(s+)$ channel massive. Regarding the $(s-)$ channel, the most important contribution due to $H_{\alpha \mathrm{FS}}^{(1)}$ comes from

$$
H_{\alpha \mathrm{FS}}^{(1)} \simeq-\frac{f}{(\pi a)^{2}} \int d x \cos \left[\sqrt{4 \pi} \theta_{c-}\right] \cos \left[\sqrt{4 \pi} \theta_{s-}\right] .
$$

Noting that we have chosen $\theta_{c-}=0$, we can again employ Majorana refermionization. The only but important effect of this contribution then consists of a renormalization of the masses $m_{1,2}$,

$$
m_{1} \rightarrow\left(m_{b}-m_{f}\right), \quad m_{2} \rightarrow m_{f},
$$

which breaks the self-duality in the $(s-)$ sector and thus drives the second Ising model off criticality as well. Majorana mean-field theory yields

$$
m_{f} \simeq(f / b) m_{b}
$$

and the associated temperature scale $T_{f} \approx(f / b) T_{b}$. Therefore both the Majorana fermion $\xi_{2}$ and the $(s+)$ field acquire the (generally small) mass $m_{f}$ due to the forward scattering contribution. At the emerging $T=0$ strong-coupling fixed point, we have long-range order in the operators

$$
\cos \left[\sqrt{\pi} \theta_{s+}\right], \quad \cos \left[\sqrt{\pi} \theta_{c-}\right], \quad \sin \left[\sqrt{\pi} \phi_{s-}\right],
$$

with exponential decay in all other operators except those of the critical $(c+)$ sector. That the first two operators become long-ranged is a direct consequence of the pinning condition $\theta_{c-}=0$. That $\sin \left[\sqrt{\pi} \phi_{s-}\right]$ exhibits longrange order can be easily understood from Eq. (5.13), since the second Ising model is now also above criticality. Therefore both $\left\langle\mu_{1}\right\rangle$ and $\left\langle\mu_{2}\right\rangle$ are finite, but $\left\langle\sigma_{1}\right\rangle=\left\langle\sigma_{2}\right\rangle=0$.

This analysis for a general interaction potential predicts that the exponents corresponding to the first (intermediate) strong-coupling point should be observable on temperature scales $T_{f}<T<T_{b}$, with a crossover to a regime $T<T_{f}$ dominated by the true $T=0$ fixed point. For long-ranged interactions, we have $T_{f} \approx T_{b}$, and the intermediate fixed point and the associated crossover phenomenon are not observable. However, for screened interactions, $T_{b}$ can be significantly larger than $T_{f}$. Finally, in the regime $T>T_{b}$, which also includes the temperature range experimentally studied in Ref. [18], the physical behavior emerging from our analysis is best characterized as that of a Luttinger liquid with an additional flavor index. In this regime it is justified to neglect the nonlinearities associated with the coupling constants $b$ and $f$. Nanotubes therefore constitute a realization of the Luttinger liquid at sufficiently high temperatures.

\section{SUSCEPTIBILITIES AND CORRELATION FUNCTIONS}

With the strong-coupling solution of Sec. V at hand, we can now examine temperature-dependent susceptibilities and other experimentally accessible quantities. Due to the Mermin-Wagner theorem, there can be no ordered state in a $1 \mathrm{D}$ system [63]. It is therefore customary to characterize the physical behavior by the most slowly decaying correlation function, which in turn indicates incipient instabilities. We have investigated correlators of charge-density wave (CDW), spin-density wave (SDW), and superconducting (SC) type. The results reported in this section are summarized in Table 1 .

One has to carefully distinguish the three temperature regimes discussed in Sec. V, since different instabilities emerge in different temperature ranges. Furthermore, we also have to distinguish the spatial oscillation period of the correlations. It is apparent from the dispersion relation in Fig. 目 that the wavelengths

$$
\lambda=\pi / k_{F}, \quad \pi / q_{F}, \quad \pi /\left(k_{F} \pm q_{F}\right)
$$

could occur in a doped armchair SWNT (we put $q_{F}>0$ for simplicity). Without doping, only the standard $\pi / k_{F}$ wavelength is found, which corresponds to an order operator effectively involving two 1D fermions at different Fermi points $\left(\alpha=-\alpha^{\prime}\right)$. In the doped case, the much longer wavelength $\pi / q_{F}$ arises if the $1 \mathrm{D}$ fermions are at the same Fermi point but move in opposite directions $\left(r=-r^{\prime}\right)$. Finally, the wavelength $\pi /\left(k_{F} \pm q_{F}\right)$ corresponds to the mixed situation $\left(\alpha=-\alpha^{\prime}, r=-r^{\prime}\right)$. The possible simultaneous occurrence of different wavelengths is a remarkable feature of doped nanotubes which has no analogue in the standard two-chain problem. This phenomenon has its ultimate origin in the unique band structure of graphite.

\section{A. CDW correlations}

In calculating the CDW correlations, we first need to find the bosonized representation of the $y$-integrated [this only amounts to a factor $2 \pi R]$ density operator $q(x)$. It can be derived from Eq. (2.5),

$q(x)=\int d y \sum_{\sigma} \Psi_{\sigma}^{\dagger}(\boldsymbol{x}) \Psi_{\sigma}(\boldsymbol{x})$ 


$$
=\sum_{p p^{\prime} \alpha \alpha^{\prime} \sigma} \int d y \varphi_{p \alpha}^{*}(\boldsymbol{x}) \varphi_{p^{\prime} \alpha^{\prime}}(\boldsymbol{x}) \psi_{p \alpha \sigma}^{\dagger}(x) \psi_{p^{\prime} \alpha^{\prime} \sigma}(x) .
$$

There is first a "slow" component $\rho(x)$ due to $p=p^{\prime}, \alpha=$ $\alpha^{\prime}$, whose bosonized form is given in Eq. (3.11). Furthermore, there is an intra-sublattice (CDW0) order parameter contributing in Eq. (6.2),

$$
\hat{O}_{C D W 0}(x) \sim \sum_{p \alpha \sigma} \psi_{p \alpha \sigma}^{\dagger}(x) \psi_{p,-\alpha, \sigma}(x) .
$$

Since the Bloch functions for different sublattices are orthogonal, one might now conclude that $p \neq p^{\prime}$ cannot give a contribution to $q(x)$. However, the corresponding finite (but generally small) matrix element allowing for a contribution due to the inter-sublattice $(\mathrm{CDW} \pi)$ order parameter

$$
\hat{O}_{C D W \pi}(x) \sim \sum_{p \alpha \sigma} \psi_{p \alpha \sigma}^{\dagger}(x) \psi_{-p, \pm \alpha, \sigma}(x)
$$

can be generated by the interactions. Such a mechanism is well-known from the study of $4 k_{F}$ components in the density operator for correlated 1D fermions 64.

To make use of the bosonized version, we then employ the unitary transformation (3.2) and find

$$
\begin{aligned}
\hat{O}_{C D W 0} & \sim \sum_{r \alpha \sigma} \widetilde{\psi}_{r \alpha \sigma}^{\dagger} \widetilde{\psi}_{r,-\alpha, \sigma} \\
& \sim \sin \left[\sqrt{\pi} \phi_{c-}+2 k_{F} x\right] \cos \left[\sqrt{\pi} \theta_{c-}\right] \\
& \times \cos \left[\sqrt{\pi} \theta_{s-}\right] \cos \left[\sqrt{\pi} \phi_{s-}\right]-(\cos \leftrightarrow \sin ) .
\end{aligned}
$$

The correlation function of $\hat{O}_{C D W 0}$ thus has the wavelength $\lambda=\pi / k_{F}$ and decays exponentially for $T<T_{b}$. In contrast, for $T>T_{b}$, its scaling dimension is $\eta=1$. We therefore omit the CDW0 mode in the sequel as it exhibits a fast subdominant decay for all temperatures.

Turning to the CDW $\pi$ correlations, we find two contributions,

$$
\begin{aligned}
\hat{O}_{1} & \sim \sum_{r \alpha \sigma}(-i r) \widetilde{\psi}_{r \alpha \sigma}^{\dagger} \widetilde{\psi}_{-r, \alpha, \sigma} \\
& \sim \cos \left[\sqrt{\pi} \theta_{c+}+2 q_{F} x\right] \cos \left[\sqrt{\pi} \theta_{c-}\right] \\
& \times \sin \left[\sqrt{\pi} \theta_{s+}\right] \sin \left[\sqrt{\pi} \theta_{s-}\right]+(\cos \leftrightarrow \sin ), \\
\hat{O}_{2} & \sim \sum_{r \alpha \sigma}(-i r) \widetilde{\psi}_{r \alpha \sigma}^{\dagger} \widetilde{\psi}_{-r,-\alpha, \sigma} \\
& \sim \cos \left[\sqrt{\pi} \theta_{c+}+2 q_{F} x\right] \cos \left[\sqrt{\pi} \phi_{c-}+2 k_{F} x\right] \\
& \times \cos \left[\sqrt{\pi} \theta_{s+}\right] \cos \left[\sqrt{\pi} \phi_{s-}\right]+(\cos \leftrightarrow \sin ) .
\end{aligned}
$$

The first operator leads to a slowly oscillating correlation function with wavelength $\lambda=\pi / q_{F}$, while the second operator implies rapid oscillations with $\lambda=\pi /\left(k_{F} \pm q_{F}\right)$. Both operators exhibit exponential decay at the lowest temperatures, $T<T_{f}$. In the intermediate temperature regime, $T_{f}<T<T_{b}, \hat{O}_{2}$ also leads to exponentially decaying correlation functions, but $\hat{O}_{1}$ has the scaling dimension $\eta=(3+2 K) / 8$. Finally, in the high-temperature Luttinger liquid regime, $T>T_{b}$, the scaling dimension $\eta=(3+K) / 4$ arises for both operators. For scaling dimension $\eta$, the equal-time correlation function is

$$
\left\langle\hat{O}_{1}(x) \hat{O}_{1}\left(x^{\prime}\right)\right\rangle \sim \cos \left[2 q_{F}\left(x-x^{\prime}\right)\right]\left|x-x^{\prime}\right|^{-2 \eta},
$$

and the corresponding susceptibility has the temperature dependence $\chi\left(2 q_{F}\right) \sim T^{2 \eta-2}$. For correlations of $\hat{O}_{2}$, instead of $2 q_{F}$ one has the wavevector $2\left(k_{F} \pm q_{F}\right)$. Since only the slowly oscillating contribution due to $\hat{O}_{1}$ exhibits power-law behavior for $T_{f}<T<T_{b}$, it is favored over the $\hat{O}_{2}$ contribution by a larger prefactor for $T>T_{b}$, at least for well-screened interactions.

\section{B. Higher-order CDW correlations}

There is also a contribution to the density correlation function effectively originating from squaring the above order parameters. Even if the original operators are irrelevant, the emerging higher-order operators can become relevant. The analogue of this behavior in the standard two-chain problem has been interpreted as incipient Wigner crystal behavior [52], since it is characterized by a wavelength $\pi / 2 k_{F}$ corresponding to the average electronic spacing. Such an interpretation is not possible in our case, since the characteristic wavevector is now $4 q_{F}$ or even $8 q_{F}$ instead of the standard value $4 k_{F}$.

The operator $\hat{O}_{C D W 0}^{2}$ stays always irrelevant, but the squared $\mathrm{CDW} \pi$ operators give important contributions. Using Eq. (5.9), we find the leading terms

$$
\begin{aligned}
\hat{O}_{1}^{2} & \sim \cos \left[\sqrt{4 \pi} \theta_{c+}+4 q_{F} x\right]\left\{-\cos \left[\sqrt{4 \pi} \theta_{s-}\right]\right. \\
& \left.+\cos \left[\sqrt{4 \pi} \theta_{c-}\right]-\cos \left[\sqrt{4 \pi} \theta_{s+}\right]\right\} \\
\hat{O}_{2}^{2} & \sim \cos \left[\sqrt{4 \pi} \theta_{c+}+4 q_{F} x\right]\left\{\cos \left[\sqrt{4 \pi} \theta_{s+}\right]\right. \\
& \left.+\cos \left[\sqrt{4 \pi} \phi_{c-}+4 k_{F} x\right]+\cos \left[\sqrt{4 \pi} \phi_{s-}\right]\right\} .
\end{aligned}
$$

Here we have kept only contributions containing the $\theta_{c+}$ field, since these are the only ones which could become relevant. Since these operators come from products of four 1D fermion operators, the Pauli principle allows exactly three terms for $\hat{O}_{1}^{2}$ and $\hat{O}_{2}^{2}$, respectively. The prefactors for the various terms in Eqs. (6.7) and (6.8) are non-universal and depend on the interactions. The scaling dimension of these operators is $\eta=1+K$ for $T>T_{b}$, so that they are always irrelevant in the Luttinger liquid regime. Furthermore, for $T<T_{b}$, we observe that $\hat{O}_{2}^{2}$ also produces only subleading contributions. In effect, we need to consider only $\hat{O}_{1}^{2}$, which indeed becomes a relevant operator for $T<T_{b}$. The scaling dimension in this temperature regime is $\eta=K$, leading to the $4 q_{F}$ oscillatory correlations 
$\left\langle\hat{O}_{C D W \pi}^{2}(x) \hat{O}_{C D W \pi}^{2}\left(x^{\prime}\right)\right\rangle \sim \cos \left[4 q_{F}\left(x-x^{\prime}\right)\right]\left|x-x^{\prime}\right|^{-2 K}$.

This turns out to be the dominant instability at $T<T_{b}$

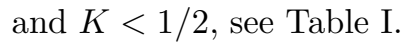

In the same line of reasoning, there also exists an effective contribution due to $\hat{O}_{C D W \pi}^{4}$. This operator is relevant for extremely strong correlations,

$$
\hat{O}_{C D W \pi}^{4} \sim \cos \left[\sqrt{16 \pi} \theta_{c+}+8 q_{F} x\right],
$$

with wavelength $\pi / 4 q_{F}$ and scaling dimension $\eta=4 K$ for all temperatures. By comparing to the scaling dimension $\eta=(3+K) / 4$ of $\hat{O}_{1}$, the $\lambda=\pi / 4 q_{F} \mathrm{CDW} \pi$ state is seen to represent the dominant incipient instability in the Luttinger liquid regime $T>T_{b}$ for very strong but still realistic correlations, $K<1 / 5$. At temperatures below $T_{b}$, the $8 q_{F}$ operator (6.10) leads to only subdominant contributions and can safely be ignored.

\section{SDW correlations}

Next we turn to spin-density wave correlations. Due to the underlying $S U(2)$ spin isotropy, it is sufficient to study the $s_{z}$ correlations which can be readily evaluated. Similar to the CDW case, we have the order parameters

$$
\begin{aligned}
& \hat{O}_{S D W 0}(x) \sim \sum_{p \alpha \sigma} \sigma \psi_{p \alpha \sigma}^{\dagger}(x) \psi_{p,-\alpha, \sigma}(x), \\
& \hat{O}_{S D W \pi}(x) \sim \sum_{p \alpha \sigma} \sigma \psi_{p \alpha \sigma}^{\dagger}(x) \psi_{-p, \pm \alpha, \sigma}(x) .
\end{aligned}
$$

Again the intra-sublattice SDW0 component can be ignored as it only leads to $\eta \geq 1$. This is apparent from the bosonized representation

$$
\begin{aligned}
\hat{O}_{S D W 0} & \sim \sum_{r \alpha \sigma} \sigma \widetilde{\psi}_{r \alpha \sigma}^{\dagger} \widetilde{\psi}_{r,-\alpha, \sigma} \\
& \sim \cos \left[\sqrt{\pi} \phi_{c-}+2 k_{F} x\right] \cos \left[\sqrt{\pi} \theta_{c-}\right] \\
& \times \cos \left[\sqrt{\pi} \theta_{s-}\right] \sin \left[\sqrt{\pi} \phi_{s-}\right]-(\cos \leftrightarrow \sin ) .
\end{aligned}
$$

The important part comes from the SDW $\pi$ order operator

$$
\hat{O}_{S D W \pi} \sim \sum_{p \alpha \sigma} \sigma \psi_{p \alpha \sigma}^{\dagger} \psi_{-p, \pm \alpha, \sigma}
$$

Rotation to the right/left-moving basis and subsequent bosonization gives the two operators

$$
\begin{aligned}
\hat{O}_{a} & \sim \sum_{r \alpha \sigma}(-i r \sigma) \widetilde{\psi}_{r \alpha \sigma}^{\dagger} \widetilde{\psi}_{-r, \alpha, \sigma} \\
& \sim \sin \left[\sqrt{\pi} \theta_{c+}+2 q_{F} x\right] \cos \left[\sqrt{\pi} \theta_{c-}\right] \\
& \times \cos \left[\sqrt{\pi} \theta_{s+}\right] \sin \left[\sqrt{\pi} \theta_{s-}\right]+(\cos \leftrightarrow \sin ), \\
\hat{O}_{b} & \sim \sum_{r \alpha \sigma}(-i r \sigma) \widetilde{\psi}_{r \alpha \sigma}^{\dagger} \widetilde{\psi}_{-r,-\alpha, \sigma} \\
& \sim \cos \left[\sqrt{\pi} \theta_{c+}+2 q_{F} x\right] \sin \left[\sqrt{\pi} \phi_{c-}+2 k_{F} x\right] \\
& \times \cos \left[\sqrt{\pi} \theta_{s+}\right] \sin \left[\sqrt{\pi} \phi_{s-}\right]+(\cos \leftrightarrow \sin ) .
\end{aligned}
$$

Both operators lead to exponentially decaying correlations at $T<T_{f}$. Furthermore, in the regime $T_{f}<T<$ $T_{b}, \hat{O}_{a}$ has the same scaling dimension $\eta=(3+2 K) / 8$ as the CDW $\pi$ operator $\hat{O}_{1}$ in Eq. (6.4), with exponential decay in $\hat{O}_{b}$. Finally, for $T>T_{b}$, both $\hat{O}_{a, b}$ have again the same scaling dimension $\eta=(3+K) / 4$ as $\hat{O}_{1,2}$. We then arrive at exactly the same power laws for SDW and CDW correlations.

These power laws represent the most slowly decaying correlations of the system in the wide temperature range $T>T_{f}$, provided the correlations are not too strong, see Table i. To discriminate among CDW and SDW correlations, we have to study prefactors of the power laws. From Eqs. (6.4) and (6.12), since $\cos \left[\sqrt{\pi} \theta_{s+}\right]$ appears in $\hat{O}_{a}$ instead of $\sin \left[\sqrt{\pi} \theta_{s+}\right]$ for $\hat{O}_{1}$, and because at temperatures $T<T_{f}$ we have the pinning condition $\theta_{s+}=0$, see Eq. (5.16), the SDW amplitude should be larger than the CDW amplitude also for $T>T_{f}$. The magnetic $S D W$ correlations then represent the dominant instability of the nanotube in the temperature regime $T>T_{f}$ for moderate correlation strength. Remarkably, they are characterized by the simultaneous presence of the wavelengths $\lambda=\pi / q_{F}$ and $\pi /\left(k_{F} \pm q_{F}\right)$.

Unfortunately, since the intermediate temperature regime is absent for externally unscreened interactions, i.e., $T_{f} \approx T_{b}$, it appears to be rather difficult to decide which wavelength will eventually be more important for the experimental setup of Ref. [18]. For a well-screened interaction, based on the above discussion, we expect that the slow wavelength is more important. In that case, since $\hat{O}_{S D W \pi}$ can give a contribution to the $1 \mathrm{D}$ spin density $s_{z}(x)$ via the mechanism discussed in Sec. VIA, the $s_{z}$ correlations are dominated by the $2 q_{F}$ oscillatory part,

$$
\left\langle s_{z}(x) s_{z}\left(x^{\prime}\right)\right\rangle \sim \cos \left[2 q_{F}\left(x-x^{\prime}\right)\right]\left|x-x^{\prime}\right|^{-2 \eta} .
$$

For the distances $\left|x-x^{\prime}\right|$ where the correlations are not already vanishingly small, the cosine factor stays essentially at unity. Hence the SDW correlations show a pronounced ferromagnetic character. This reasoning offers an explanation for the ferromagnetic tendencies observed in Ref. [18]. Our explanation could be experimentally checked by increasing the doping and hence $q_{F}$. Eventually, antiferromagnetism should be recovered for sufficiently strong doping.

\section{Superconductivity}

Predominant superconducting (SC) features have been predicted recently in two-chain models despite the repulsive nature of the Coulomb interaction [41,49, 52]. For the nanotube, the inter-sublattice singlet and all triplet pairing operators cause irrelevant terms and therefore play no role. The dominant contribution then comes from the intra-sublattice singlet pairing ( $\mathrm{SC} 0$ ) operator 


$$
\hat{O}_{S C 0} \sim \sum_{p \alpha \sigma} \sigma \psi_{p \alpha \sigma} \psi_{p-\alpha-\sigma} .
$$

This gives the bosonized form

$$
\begin{aligned}
\hat{O}_{S C 0} & \sim \sum_{r \alpha \sigma} \sigma \widetilde{\psi}_{r \alpha \sigma} \widetilde{\psi}_{-r,-\alpha,-\sigma} \\
& \sim \cos \left[\sqrt{\pi} \phi_{c+}\right] \cos \left[\sqrt{\pi} \theta_{c-}\right] \\
& \times \cos \left[\sqrt{\pi} \theta_{s+}\right] \sin \left[\sqrt{\pi} \phi_{s-}\right]-(\cos \leftrightarrow \sin ) .
\end{aligned}
$$

For $T>T_{b}$, this operator has scaling dimension $\eta=(3+$ $1 / K) / 4$ and is therefore irrelevant. In the intermediate temperature regime $T_{f}<T<T_{b}$, we find $\eta=(3+$ $2 / K) / 8$, and $\hat{O}_{S C 0}$ is only subleading. However, for $T<$ $T_{f}$, we obtain

$$
\left\langle\hat{O}_{S C 0}(x) \hat{O}_{S C 0}\left(x^{\prime}\right)\right\rangle \sim\left|x-x^{\prime}\right|^{-1 / 2 K} .
$$

Therefore singlet superconductivity becomes the dominant instability at extremely low temperatures and for externally screened interactions with $K>1 / 2$. In practice, superconducting correlations are thus of little importance in carbon nanotubes.

\section{E. Tunneling Density of States}

Under typical experimental conditions, the contact between a SWNT and the attached Fermi-liquid leads is not always adiabatic. For nonadiabatic contacts, the physics of the conductance is related to single-electron tunneling into the nanotube, which in turn is governed by the tunneling density of states (TDOS). A similar situation also arises in tunneling microscope experiments. Here the electron tunneling occurs close to the impurity position, the role of the impurity being played by the probe itself. Therefore let us now discuss the TDOS of a SWNT. The presence of an open boundary, either due to the finite length of the nanotube or due to a strong impurity, can alter correlation functions and, in particular, modify the anomalous exponents [33]. Hence, in calculating the TDOS close to the end of the SWNT, we have to employ the open boundary bosonization method. The latter can be developed for nanotubes in full analogy to spin chains [65] and quantum wires [35]. Here we shall give the main ideas of the approach but avoid technical details, which the interested reader can find in Refs. [35, 65].

For simplicity, let us consider a semi-infinite nanotube, $x>0$. The left-moving electrons in the nanotube then undergo perfect reflection at $x=0$, where they are transformed into a right-mover going back to $x \rightarrow \infty$. Mathematically, the perfect reflection leads to boundary conditions imposed on the right- and left-moving electron field operators at $x=0$,

$$
\widetilde{\psi}_{+\alpha \sigma}(0)=\exp \left(i \delta_{0}\right) \widetilde{\psi}_{-\alpha \sigma}(0) \text {. }
$$

Here $\delta_{0}$ is a scattering phase shift, which depends on the shape-confining potential. For example, if the end of the nanotube has dangling (but hydrogen-saturated) bonds, $\delta_{0}$ is different compared to a closing cap, i.e., if the nanotube closes into a fullerene half-sphere. However, this phase shift is of no further interest here and can be chosen as zero by shifting the position of the boundary 35.

Due to the boundary condition (6.16), the right- and left-movers are not independent fields anymore. It therefore makes sense to switch to a description in terms of only a right-moving field which is now defined for all $x$,

$$
\Psi_{\alpha \sigma}(x)= \begin{cases}\widetilde{\psi}_{+\alpha \sigma}(x) & (x>0), \\ \widetilde{\psi}_{-\alpha \sigma}(-x) & (x<0) .\end{cases}
$$

The advantage of this representation is that the rightmoving field operator $\Psi_{\alpha \sigma}$, being defined on the infinite interval, can be standardly bosonized,

$$
\Psi_{\alpha \sigma}(x)=\frac{1}{\sqrt{2 \pi a}} \exp \left[i \varphi_{\alpha \sigma}(x)\right],
$$

where $\varphi_{\alpha \sigma}$ is a chiral Bose field. For brevity, we have omitted the Klein factors and the oscillating spatial exponent. Both cancel out in what follows. It is then helpful to define the chiral fields

$$
\begin{aligned}
& \Phi_{c+}=\frac{1}{4 \sqrt{\pi}} \sum_{\alpha \sigma} \varphi_{\alpha \sigma}, \\
& \Phi_{c-}=\frac{1}{4 \sqrt{\pi}} \sum_{\alpha \sigma} \alpha \varphi_{\alpha \sigma}, \\
& \Phi_{s+}=\frac{1}{4 \sqrt{\pi}} \sum_{\alpha \sigma} \sigma \varphi_{\alpha \sigma}, \\
& \Phi_{s-}=\frac{1}{4 \sqrt{\pi}} \sum_{\alpha \sigma} \alpha \sigma \varphi_{\alpha \sigma} .
\end{aligned}
$$

In terms of these fields, Eq. (6.18) takes the form

$$
\Psi_{\alpha \sigma}=\frac{1}{\sqrt{2 \pi a}} \exp \left\{i \sqrt{\pi}\left[\Phi_{c+}+\alpha \Phi_{c-}+\sigma \Phi_{s+}+\alpha \sigma \Phi_{s-}\right]\right\} .
$$

If electron-electron interactions are neglected, the $\Phi_{j \delta}$ are free chiral fields with the Hamiltonian

$$
\begin{aligned}
& H_{0}=-i \sum_{r \alpha \sigma} r \int_{0}^{\infty} d x \widetilde{\psi}_{r \alpha \sigma}^{\dagger} \partial_{x} \widetilde{\psi}_{r \alpha \sigma}= \\
& -i \sum_{\alpha \sigma} \int_{-\infty}^{\infty} d x \Psi_{\alpha \sigma}^{\dagger} \partial_{x} \Psi_{\alpha \sigma}=v \sum_{j \delta} \int d x\left(\partial_{x} \Phi_{j \delta}\right)^{2} .
\end{aligned}
$$

In the presence of interactions, however, the nonlocal representation (6.17) causes nonlocal interaction terms of the form $\rho(x) \rho(-x)$ such that the electron densities are coupled at mirror-imaged points. Nevertheless, this interaction remains quadratic in the Bose field and can be 
diagonalized. Following the steps of Ref. [35], the appropriate Bogoliubov rotation is

$$
\Phi_{c+}(x) \rightarrow c \Phi_{c+}(x)-s \Phi_{c+}(-x),
$$

where $c=\cosh \phi_{0}$ and $s=\sinh \phi_{0}$ parametrize the Luttinger liquid exponent $K=\exp \left(2 \phi_{0}\right)$.

Two remarks are in order here. (1) We only consider the Luttinger liquid phase $T>T_{b}$ in the following. Then the only important interaction term is $H_{\alpha \mathrm{FS}}^{(0)}$. In the lowtemperature massive phases, the single-electron TDOS develops a gap, but there could still be gapless higherorder multi-electron scattering processes. (2) We consider the zero-momentum limit for the charge exponent $K$, i.e., distances (times) larger than the Coulomb screening length. The behavior of the correlation functions for distances closer to the boundary than the screening length is fairly complex, see Appendix C of Ref. [35].

Combining Eqs. (6.19) and (6.21), we obtain the main formula of the open boundary bosonization,

$$
\begin{aligned}
& \Psi_{\alpha \sigma}(x)=\frac{1}{\sqrt{2 \pi a}} \exp \left\{i \sqrt { \pi } \left[c \Phi_{c+}(x)-s \Phi_{c+}(-x)\right.\right. \\
& \left.\left.+\alpha \Phi_{c-}(x)+\sigma \Phi_{s+}(x)+\alpha \sigma \Phi_{s-}(x)\right]\right\} .
\end{aligned}
$$

It follows that close to the boundary, i.e., for $\max (x, y) \ll$ $v t$, the single-electron Greens function is of the form

$$
\left\langle\Psi^{\dagger}(x, t) \Psi(y, 0)\right\rangle \sim t^{-(1 / K+3) / 4} .
$$

The boundary scaling dimension of the electron field operator is therefore

$$
\bar{\Delta}=\frac{1}{8 K}+\frac{3}{8},
$$

as opposed to its bulk scaling dimension,

$$
\Delta=\frac{1}{16}\left(\frac{1}{K}+K\right)+\frac{3}{8} .
$$

Making use of the text-book definition of the density of states as the imaginary part of the single-electron Greens function, we obtain for the TDOS at the end of the nanotube,

$$
\rho_{\mathrm{end}}(\omega) \sim \omega^{2 \bar{\Delta}-1}
$$

which is different from the bulk density of states

$$
\rho_{\text {bulk }}(\omega) \sim \omega^{2 \Delta-1} .
$$

Since $\bar{\Delta}>1 / 2$ for $K<1$, the TDOS always vanishes with the frequency (or the temperature) approaching zero, similar to the situation encountered in quantum wires [33]. For a Fermi liquid, we would instead have $\Delta=\Delta=1 / 2$, and the exponents in Eqs. (6.26) and (6.27) are both zero. For the $(10,10)$ nanotube of Ref.
[18], taking our previous estimate $K \simeq 0.18$, the exponents in Eqs. (6.26) and (6.27) are 1.13 and 0.46, respectively. Therefore single-electron tunneling is strongly suppressed close to the end of the nanotube. Finally, we stress again that these results are also valid in the vicinity of a strong impurity. At low energy scales, the SWNT is effectively cut into two independent parts because of the impurity, see Sec. VII.

\section{TRANSPORT AND CONDUCTANCE LAWS}

In this section, we discuss transport through a SWNT. Since non-Fermi liquid laws are only pronounced if contact resistances between the attached leads and the nanotube are smaller than $\approx h / e^{2}$, we assume good contact to the transport voltage sources. Since there are four transport channels $(j \delta)$, a SWNT adiabatically connected to external leads exhibits the perfect conductance $G=G_{0}=4 e^{2} / h$ in the absence of impurity backscattering. Even in the presence of weak backscattering, $G$ approaches $G_{0}$ at sufficiently high temperatures.

The effect of impurities on the conductance can be very pronounced in a Luttinger liquid [33]. In a SWNT, there are several possible sources for impurities as discussed in the Introduction. The dominant coupling to the impurity is due to the CDW $\pi$ operators $\hat{O}_{1}$ and $\hat{O}_{2}$ in Eqs. (6.4) and (6.5). Since both operators have essentially the same effect, we focus on $\hat{O}_{1}$ and get the form

$$
\begin{aligned}
H_{\mathrm{imp}} & =\int d x m(x)\left\{\cos \left[\sqrt{\pi} \theta_{c+}+2 q_{F} x\right] \cos \left[\sqrt{\pi} \theta_{c-}\right]\right. \\
& \left.\times \sin \left[\sqrt{\pi} \theta_{s+}\right] \sin \left[\sqrt{\pi} \theta_{s-}\right]+(\cos \leftrightarrow \sin )\right\} .
\end{aligned}
$$

From the perturbation series in $m(x)$ for the partition sum, one can check that the higher-order operators of Sec. VIB are implicitly contained in Eq. (7.1). There is no need to explicitly take them into account in $H_{\text {imp }}$.

Let us analyze the consequences of this Hamiltonian for the simplest case of a single pointlike impurity, $m(x)=$ $m \delta(x)$. We assume an impurity without any internal dynamics, e.g., a frozen twist of the nanotube. The conductance corrections $\delta G$ defined by $G=G_{0}-\delta G$ depend on the temperature regime under study. Perturbation theory yields in order $m^{2}$ the power laws

$$
\begin{aligned}
\delta G & \sim T^{(K-1) / 2} \quad\left(T>T_{b}\right), \\
& \sim T^{(2 K-5) / 4} \quad\left(T_{f}<T<T_{b}\right) .
\end{aligned}
$$

At very low temperatures, $T<T_{f}$, one is in the strongcoupling regime and perturbation theory does not apply anymore. In fact, we find that the conductance is totally suppressed at zero temperature. In the regime $T<T_{f}$, the average value of the impurity operator (7.1) vanishes, while its correlation function decays exponentially in time. At first sight, the impurity has therefore 
no effect. However, it may still generate a relevant operator. In order to see how this happens, we calculate the second-order correction to the action due to the local impurity operator,

$$
\begin{aligned}
\delta S^{2} & \sim m^{2} \int d \tau \int d \tau^{\prime} \exp \left[-\gamma\left|\tau-\tau^{\prime}\right|\right] \\
& \times \cos \left[\sqrt{\pi} \theta_{c+}(0, \tau)\right] \cos \left[\sqrt{\pi} \theta_{c+}\left(0, \tau^{\prime}\right)\right]
\end{aligned}
$$

where $\gamma \sim m_{f}$ characterizes the decay rate of correlations of the gapped degrees of freedom involved in the impurity operator (7.1). Since we are interested in the low-energy (long-time) behavior of the system, we are eligible to contract the $\tau$ and $\tau^{\prime}$ variables in the above integration. Then, using the operator identity (5.9), the total charge field satisfies the effective Hamiltonian

$$
H_{\mathrm{eff}}\left[\theta_{c+}\right]=H_{0}\left[\theta_{c+}\right]+\frac{\Lambda}{\pi a} \cos \left[\sqrt{4 \pi K} \theta_{c+}(0)\right],
$$

where the effective impurity strength is estimated to be $\Lambda \sim m^{2} / m_{f}$, and we have rescaled $\theta_{c+} \rightarrow \sqrt{K} \theta_{c+}$. Of course, the exponent found in Eq. (7.5) below is independent of $\Lambda$. Note that the result (7.4) also follows from Eq. (6.7) for $T<T_{f}$ by virtue of the pinning conditions discussed in Sec. V.

The effective Hamiltonian (7.4) is now identical to the one describing an impurity in a spinless Luttinger liquid. The reasoning of Kane and Fisher [33] therefore fully applies and yields the low-temperature conductance

$$
G \sim T^{-2+2 / K}
$$

This implies a total suppression of transport through the nanotube at $T=0$ in the presence of a single, arbitrarily weak impurity.

Finally, we mention that Luttinger liquid behavior could also be observed in experiments on crossed nanotubes. Nonequilibrium transport through crossed Luttinger liquids has been studied theoretically in Ref. [66]. Following this analysis, a distinct nonlinear dependence of the current through one SWNT $(j=1)$ on the cross voltage applied to the other $\operatorname{SWNT}(j=2)$ can be expected. Assuming a point-like contact between both nanotubes at $x=0$, the only important coupling mechanism is of electrostatic nature,

$$
H_{c}=\widetilde{\lambda} \rho_{1}(0) \rho_{2}(0)
$$

which becomes relevant if the scaling dimension $\eta$ of the individual local density operators $\rho_{j=1,2}(0)$ satisfies $\eta<1 / 2$. This condition can only be met by the higherorder CDW $\pi$ contributions in Sec.VIB. In the Luttinger liquid regime $T>T_{b}$, the leading operator is $\hat{O}_{C D W \pi}^{4}$ in Eq. 6.10), with $\eta=4 K$. The coupling (7.6) is then irrelevant unless the condition $K<1 / 8$ is fulfilled. According to Eq. (3.23), such strong correlations could be achieved by studying sufficiently long nanotubes. Furthermore, at lower temperatures $T<T_{b}$, the dominant coupling comes from $\hat{O}_{1}^{2}$ in Eq. (6.7), with $\eta=K$. Therefore the relevancy condition is now less stringent, $K<1 / 2$, and the effects predicted in Ref. [66] should show up already for short nanotubes at sufficiently low temperatures.

\section{IMPURITY SCREENING}

In this section, we study the electronic screening cloud induced by an impurity sitting at, say, $x=0$. This could be a substitutional atom replacing one of the carbon atoms on the tube surface (like B or N), an intercalating atom (like $\mathrm{Na}$ ) sitting inside the nanotube at some radius $r<R$, or an incident positron. The (externally unscreened) Fourier transformed potential for impurity charge $Z e$ is 38

$$
\widetilde{V}_{\mathrm{imp}}(q)=4 \pi Z e^{2} I_{0}(q r) K_{0}(q R),
$$

with the modified Bessel functions $I_{0}$ and $K_{0}$ 46. The small- $q$ response of the $\pi$ electrons to this perturbation can be computed in an exact manner by linear screening, and the RPA treatment and the corresponding results of Ref. 38 fully apply. However, for the finite wavevectors listed in Eq. (6.1), linear screening is known to break down in one dimension 34 37. The 1D Friedel oscillation cannot be computed by an RPA-like treatment.

The impurity strength $m$ determining the amplitude of the Friedel oscillation is given by $\widetilde{V}_{\text {imp }}\left(2 q_{F}\right)$ [for wavelength $\left.\lambda=\pi / q_{F}\right]$, or $\widetilde{V}_{\mathrm{imp}}\left(2 k_{F}\right)$ [for $\lambda=\pi / k_{F} \simeq$ $\left.\pi /\left(k_{F} \pm q_{F}\right)\right]$. In the latter case, $m$ is strongly dependent on the position of the impurity. If the impurity sits in the center of the nanotube $(r=0)$, we get exponential suppression of $m$ due to Eq. (8.1). This implies a strongly reduced amplitude of the Friedel oscillation. However, the actual value of $m$ does not affect the power laws reported below. These are universal, i.e., independent of strength, position $r$, or nature of the impurity.

As discussed in Sec. VIA, the 1D density operator $q(x)$ has several contributions. The expectation value of the slow part $\rho(x)$ in the presence of the impurity can be obtained by RPA 38 and is not further discussed here. The contributions beyond $\rho(x)$ are due to the CDW order operators in Sec. V1. These arise from mixing the different types of 1D fermion operators.

Following Ref. [34, it is straightforward to extract the power law decay of the Friedel oscillation. For $T>T_{b}$, the CDW $\pi$ operators $\hat{O}_{1}$ and $\hat{O}_{2}$ specified in Eqs. 6.4 and (6.5) lead to the simultaneous presence of the wavelengths $\pi / q_{F}$ and $\pi /\left(k_{F} \pm q_{F}\right)$. Contrary to the RPA analysis [38], our exact treatment of Coulomb interactions yields for $x \gg a$ 34.67,

$$
\begin{array}{rlrl}
\left\langle q_{2 q_{F}}(x)\right\rangle & \sim \cos \left[2 q_{F} x\right]\left(x / x_{0}\right)^{-(3+K) / 4} & & \left(x \gg x_{0}\right) \\
& \sim \cos \left[2 q_{F} x\right]\left(x / x_{0}\right)^{-(1+K) / 2} & \left(x \ll x_{0}\right),
\end{array}
$$




$$
\begin{array}{rlr}
\left\langle q_{2 k_{F}}(x)\right\rangle & \sim \cos \left[2\left(k_{F} \pm q_{F}\right) x\right]\left(x / x_{0}\right)^{-(3+K) / 4} & \left(x \gg x_{0}\right) \\
& \sim \cos \left[2\left(k_{F} \pm q_{F}\right) x\right]\left(x / x_{0}\right)^{-(1+K) / 2} & \left(x \ll x_{0}\right)
\end{array}
$$

We omit possible phase shifts here. Furthermore, $x_{0}$ sets the appropriate length scale, which can be different for the various oscillation periods since it depends on the impurity strength, $x_{0} \sim m^{-4 /(1-K)}$. The asymptotic exponents for $x \gg x_{0}$ can be obtained from open boundary bosonization, see Sec.VIE, while the behavior close to the impurity follows from perturbation theory in $m$. Here the important point is that the Friedel oscillation is always slower than the standard $1 / x$ Fermi liquid result. Furthermore, the decay becomes slower as the impurity is approached. Following the reasoning of Ref. [36], the slow decay of the Friedel oscillation is the physical reason for the conductance suppression by a single impurity discussed in Sec.VII. Interestingly, we have again several different oscillation periods, namely a slow one, $\lambda=\pi / q_{F}$, which is superimposed onto rapid oscillations with wavelength $\lambda=\pi /\left(k_{F} \pm q_{F}\right)$. The Friedel oscillations induced by a strong impurity or by the ends of the nanotube can be quite pronounced and should be detectable by placing a STM tip close to the nanotube.

The power laws (8.2) and (8.3) are modified due to the $8 q_{F}$ operator (6.10) in the case of extremely strong correlations. This operator plays no role at temperatures $T<T_{b}$, but in the Luttinger liquid regime it becomes dominant as soon as $K<1 / 5$. Instead of Eqs. 8.2 and (8.3), we then find the wavelength $\pi / 4 q_{F}$,

$$
\begin{array}{rlrl}
\left\langle q_{8 q_{F}}(x)\right\rangle & \sim \cos \left[8 q_{F} x\right]\left(x / x_{0}\right)^{-4 K} & & \left(x \gg x_{0}\right) \\
& \sim \cos \left[8 q_{F} x\right]\left|\ln \left(x / x_{0}\right)\right| & \left(x \ll x_{0}\right) .
\end{array}
$$

The only logarithmically slow decay of the Friedel oscillation close to the impurity follows from Ref. [68].

The behavior of the Friedel oscillations discussed so far applies only to the "Luttinger liquid" regime, $T>$ $T_{b}$. In the intermediate temperature regime, $T_{f}<T<$ $T_{b}$, and for not too strong correlations, $1 / 2<K<1$, the exponents in these power laws become $-(3+2 K) / 8$ for $x \gg x_{0}$, and $(1-2 K) / 4$ for $x \ll x_{0}$, respectively. For strong correlations, $K<1 / 2$, the squared CDW $\pi$ operators of Sec. VIB are more important than $\hat{O}_{1}$ and $\hat{O}_{2}$. In that case, only the slow oscillation period $\pi / 2 q_{F}$ corresponding to Eq. (6.7) shows up, and we obtain

$$
\begin{array}{rlrl}
\left\langle q_{4 q_{F}}(x)\right\rangle & \sim \cos \left[4 q_{F} x\right]\left(x / x_{0}\right)^{-K} & \left(x \gg x_{0}\right) \\
& \sim \cos \left[4 q_{F} x\right]\left|\ln \left(x / x_{0}\right)\right| \quad\left(x \ll x_{0}\right) .
\end{array}
$$

Finally, at the lowest temperatures, $T<T_{f}$, the same result is found for $K<1 / 2$. For $K>1 / 2$, the perturbative exponent $\sim x^{1-2 K}$ applies to the short-distance behavior, with the same asymptotic exponent as in Eq. (8.5).

The overall behavior of Friedel oscillations in nanotubes is considerably more complicated than predicted by linear screening. We find several different slow algebraic power laws in various regions of parameter space. (8.31)hey are all slower than the standard $1 / x$ decay. Furthermore, there exist different wavelengths for the respective dominant contributions. If measurements of the charge screening cloud become feasible, one could extract the important correlation parameter $K$ and directly observe the various regions of the phase diagram corresponding to Table if from the Friedel oscillation.

\section{CONCLUSIONS}

In this work we have formulated and analyzed the effective low-energy theory of isolated single-wall carbon nanotubes. Long and clean nanotubes have already been fabricated, and we believe that the non-Fermi liquid effects discussed here will be observed in the near future.

In fact, there is some evidence that one of these effects, namely the predominance of ferromagnetic correlations, has already been observed in Ref. [18]. Other signatures of non-Fermi-liquid behavior include anomalous interaction-dependent power laws for the conductance or the tunneling density of states. Nanotubes are promising candidates for revealing the Luttinger liquid behavior generally expected for one-dimensional metals. According to our theory, at temperatures above the scale $T_{b}$ (where $T_{b} \approx 0.1 \mathrm{mK}$ for the setup of Ref. [18]), a nanotube behaves as a spin- $\frac{1}{2}$ Luttinger liquid with an additional flavor index. At lower temperatures, more complicated phases characterized by gaps in the neutral modes emerge.

A special feature of doped nanotubes is the simultaneous occurrence of different wavelengths for the various order parameter correlations. The doping is characterized by a small wavevector $q_{F} \ll k_{F}$, and one quite generally finds pronounced instabilities for the long wavelengths $\lambda=\pi / q_{F}, \pi / 2 q_{F}$ and $\pi / 4 q_{F}$, besides the short wavelength $\lambda=\pi /\left(k_{F} \pm q_{F}\right)$. Another interesting aspect of nanotubes is the length $(L)$ dependence of their properties. For instance, considering a SWNT with sufficiently small $L$, our continuum approach for explaining ferromagnetic tendencies should eventually be replaced by a Hund's rule applying to the "molecule". In our opinion, this transition from a molecule to the "solid-state" limit of large $L$ deserves further attention.

The physics of nanotubes is likely to reveal further surprises in the future. Besides the exciting prospect of finding Luttinger liquid behavior in a potentially very clean way, nanotubes might act as basic elements for molecular electronics devices, e.g., as highly conducting wires. Another line of development could employ networks of nanotubes, where novel correlation effects can be anticipated. We hope that our theory will be useful in addressing and resolving these issues. 


\section{ACKNOWLEDGMENTS}

We wish to thank C. Dekker, M.H. Devoret, H. Grabert, C.L. Kane and A.A. Nersesyan for inspiring discussions, and acknowledge financial support by the EPSRC of the United Kingdom.

[1] T.W. Ebbesen, Physics Today 49(6), 26 (1996); R.E. Smalley, Rev. Mod. Phys. 69, 723 (1997); P.G. Collins, A. Zettl, H. Bando, A. Thess, and R.E. Smalley, Science 278, 100 (1997).

[2] S. Iijima, Nature 354, 56 (1991).

[3] N. Hamada, S. Sawada, and A. Oshiyama, Phys. Rev. Lett. 68, 1579 (1992).

[4] J.W. Mintmire, B.I. Dunlap, and C.T. White, Phys. Rev. Lett. 68, 631 (1992).

[5] R. Saito, M. Fujita, G. Dresselhaus, and M.S. Dresselhaus, Appl. Phys. Lett. 60, 2204 (1992).

[6] R. Saito, M. Fujita, G. Dresselhaus, and M.S. Dresselhaus, Phys. Rev. B 46, 1804 (1992).

[7] H. Dai, E.W. Wong, and C.M. Lieber, Science 272, 523 (1996).

[8] T.W. Ebbesen, H.J. Lezec, H. Hiura, J.W. Bennett, H.F. Ghaemi, and T. Thip, Nature 382, 54 (1996).

[9] A.Yu. Kasumov, I.I. Khodos, and C. Colliex, Europhys. Lett. 34, 429 (1996).

[10] A. Thess, R. Lee, P. Nikolaev, H. Dai, P. Petit, C. Xu, Y.H. Lee, S.G. Kim, A.G. Rinzler, D.T. Colbert, G.E. Scuseria, D. Tomanek, J.E. Fischer, and R.E. Smalley, Science 273, 483 (1996).

[11] J.W.G. Wildöer, L.C. Venema, A.G. Rinzler, R.E. Smalley, and C. Dekker, Nature 391, 59 (1998); T.W. Odom, J.L. Huang, P. Kim, and C.M. Lieber, ibid. 391, 59 (1998).

[12] C. Journet, W.K. Maser, P. Bernier, A. Loiseau, M.L. de la Chapelle, S. Lefrant, P. Deniard, R. Lee, and J.E. Fischer, Nature 388, 756 (1997).

[13] L. Langer, V. Bayot, E. Grivei, J.-P. Issi, J.P. Heremans, C.H. Olk, L. Stockman, C. van Haesendonck, and Y. Bruynseraede, Phys. Rev. Lett. 76, 479 (1996).

[14] J.E. Fischer, H. Dai, A. Thess, R. Lee, N.M. Hanjani, D.L. Dehaas, and R.E. Smalley, Phys. Rev. B 55, R4921 (1997).

[15] M. Bockrath, D.H. Cobden, P.L. McEuen, N.G. Chopra, A. Zettl, A. Thess, and R.E. Smalley, Science 275, 1922 (1997).

[16] Single Charge Tunneling, edited by H. Grabert and M.H. Devoret, NATO ASI Ser. B, Vol. 294 (Plenum, New York, 1992).

[17] D. Pines and Ph. Nozières, The Theory of Quantum Liquids (Benjamin, New York, 1966).

[18] S.J. Tans, M.H. Devoret, H. Dai, A. Thess, R.E. Smalley, L.J. Geerligs, and C. Dekker, Nature 386, 474 (1997); S.J. Tans, M.H. Devoret, R.J.A. Groeneveld, and C. Dekker, preprint.
[19] G. Grüner, Density Waves in Solids (Addison-Wesley, Reading, 1994).

[20] X.G. Wen, Phys. Rev. B 44, 5708 (1991).

[21] A.O. Gogolin, Ann. Phys. (Paris) 19, 411 (1994).

[22] J. Voit, Rep. Prog. Phys. 57, 977 (1995).

[23] A.O. Gogolin, A.A. Nersesyan, and A.M. Tsvelik, Bosonization and Strongly Correlated Systems (Cambridge University Press, 1998).

[24] R. Egger and A.O. Gogolin, Phys. Rev. Lett. 795082 (1997).

[25] P.R. Wallace, Phys. Rev. 71, 622 (1947).

[26] G.S. Painter and D.E. Ellis, Phys. Rev. B 1, 4747 (1970).

[27] R.A. Jishi, D. Inomata, K. Nakao, M.S. Dresselhaus, and G. Dresselhaus, J. Phys. Soc. Jpn. 63, 2252 (1994).

[28] X. Blase, L.X. Benedict, E.L. Shirley, and S.G. Louie, Phys. Rev. Lett. 72, 1878 (1994).

[29] O.M. Yevtushenko, G.Ya. Slepyan, S.A. Maksimenko, A. Lakhtakia, and D.A. Romanov, Phys. Rev. Lett. 79, 1102 (1997).

[30] L. Chico, V.H. Crespi, L.X. Benedict, S.G. Louie, and M.L. Cohen, Phys. Rev. Lett. 76, 971 (1996).

[31] C.L. Kane and E.J. Mele, Phys. Rev. Lett. 78, 1932 (1997).

[32] D.C. Mattis, J. Math. Phys. 15, 609 (1974); X.G. Wen, Phys. Rev. B 44, 5708 (1991).

[33] C.L. Kane and M.P.A. Fisher, Phys. Rev. B 46, 15233 (1992).

[34] R. Egger and H. Grabert, Phys. Rev. Lett. 75, 3505 (1995).

[35] M. Fabrizio and A.O. Gogolin, Phys. Rev. B 51, 17827 (1995).

[36] K.A. Matveev, D. Yue, and L.I. Glazman, Phys. Rev. Lett. 71, 3351 (1993).

[37] R. Egger and H. Grabert, Phys. Rev. Lett. 79, 3463 (1997).

[38] M.F. Liu and D.S. Chuu, Phys. Rev. B 56, 4996 (1997).

[39] The extension to a semiconducting or insulating SWNT is only straightforward for the undoped case, since otherwise the gap will be opened away from the Fermi surface. A bulk mass term, however, always opens a gap directly at the Fermi surface.

[40] D.P. DiVincenzo and E.J. Mele, Phys. Rev. B 29, 1685 (1984).

[41] Yu.A. Krotov, D.H. Lee, and S.G. Louie, Phys. Rev. Lett. 78, 4245 (1997).

[42] L. Balents and M.P.A. Fisher, Phys. Rev. B 55, R11 973 (1997); ibid. 53, 12133 (1996).

[43] C.L. Kane, L. Balents, and M.P.A. Fisher, Phys. Rev. Lett. 79, 5086 (1997).

[44] E.A. Taft and H.R. Philipp, Phys. Rev. B 138, A197 (1965).

[45] I.S. Gradsteyn and I.M. Ryzhik, Table of Integrals, Series and Products (Academic Press, New York, 1980).

[46] M. Abramowitz and I.A. Stegun, Handbook of Mathematical Functions (Dover, New York, 1971).

[47] H. Yoshioka and A.A. Odintsov, unpublished results.

[48] F.V. Kusmartsev, A. Luther, and A.A. Nersesyan, JETP Lett. 55, 692 (1992).

[49] M. Fabrizio, Phys. Rev. B 48, 15838 (1993).

[50] A.M. Finkelstein and A.I. Larkin, Phys. Rev. B 47, 10461 (1993). 
[51] D.V. Khveshchenko and T.M. Rice, Phys. Rev. B 50, 252 (1994).

[52] H.J. Schulz, Phys. Rev. B 53, R2959 (1996).

[53] P. Kopietz, V. Meden, and K. Schönhammer, Phys. Rev. B 56, 7232 (1997).

[54] E. Orignac and T. Giamarchi, Phys. Rev. B 56, 7167 (1997).

[55] For numerical studies of two-leg Hubbard ladders, see R.M. Noack, N. Bulut, D.J. Scalapino, and M.G. Zacher, Phys. Rev. B 56, 7162 (1997), and references therein.

[56] P.W. Anderson, Phys. Rev. Lett. 67, 3845 (1991).

[57] X.G. Wen, Phys. Rev. B 42, 6623 (1990).

[58] C. Castellani, C. Di Castro, and W. Metzner, Phys. Rev. Lett. 69, 1703 (1992).

[59] Here the Pauli matrices act on a separate Hilbert space connected with the Klein factors and not on the $r \alpha \sigma$ quantum states.

[60] On half-filling $\left(q_{F}=0\right)$, there are two additional contributions to the bosonized Hamiltonian not specified here. The first comes from " $\alpha$ US" Umklapp processes with $\alpha_{1}=\alpha_{2}=-\alpha_{3}=-\alpha_{4}$. The second comes from $H_{\alpha \mathrm{FS}}^{(1)}$ given in Eq. (2.23), which presents another contribution beyond Eq. (3.18) on half-filling. Both terms cause a gap in the $(c+)$ sector and imply insulating behavior at energy scales below the gap.

[61] J. Cardy, Scaling and Renormalization in Statistical Physics (Cambridge University Press, 1996).

[62] C. Itzykson and J.-M. Drouffe, Statistical Field Theory, Vol. 1, Chap. 2 (Cambridge University Press, 1989).

[63] In order to avoid confusion, we note that it is a continuous symmetry which cannot be broken in 1D, while Ising-type ordering is of course allowed.

[64] H.J. Schulz, Phys. Rev. Lett. 71, 1864 (1993).

[65] S. Eggert and I. Affleck, Phys. Rev. B 46, 10866 (1992).

[66] A. Komnik and R. Egger, Phys. Rev. Lett. 80, xxx (1998).

[67] Above the thermal length scale $x_{T}=v / k_{B} T$, these power laws are cut off by an exponential decay due to thermal fluctuations. Strictly speaking, the condition $x \gg x_{0}$ should therefore read $x_{0} \ll x \ll x_{T}$.

[68] A. Leclair, F. Lesage, and H. Saleur, Phys. Rev. B 54, 13 597 (1996); F. Lesage and H. Saleur, J. Phys. A: Math. Gen. 30, L457 (1997).

TABLE I. Dominant correlations in an armchair SWNT as a function of temperature and of the correlation parameter $K<1$, with the respective scaling dimension $\eta$ and wavelength $\lambda$.

\begin{tabular}{|c|c|c|c|c|}
\hline$T$ & $K$ & |Туре & $\eta$ & $\lambda$ \\
\hline $\bar{T}>T_{b}$ & $\begin{array}{l}1 / 5<K \\
K<1 / 5\end{array}$ & $\begin{array}{l}\text { SDW } \pi \\
\text { CDW } \pi\end{array}$ & $\begin{array}{l}(3+K) / 4 \\
4 K\end{array}$ & $\begin{array}{l}\pi / q_{F}, \pi /\left(k_{F} \pm q_{F}\right. \\
\pi / 4 q_{F}\end{array}$ \\
\hline$\overline{T_{f}<T<T_{b}}$ & $\begin{array}{l}1 / 2<K \\
K<1 / 2\end{array}$ & \begin{tabular}{|l|}
$\mathrm{SDW} \pi$ \\
$\mathrm{CDW} \pi$
\end{tabular} & $\begin{array}{l}(3+2 K) / 8 \\
K\end{array}$ & $\begin{array}{l}\pi / q_{F} \\
\pi / 2 q_{F}\end{array}$ \\
\hline$\overline{T<T_{f}}$ & $\begin{array}{l}1 / 2<K \\
K<1 / 2\end{array}$ & \begin{tabular}{|l|}
$\mathrm{SC} 0$ \\
$\mathrm{CDW} \pi$
\end{tabular} & $\begin{array}{l}1 / 4 K \\
K\end{array}$ & \\
\hline
\end{tabular}




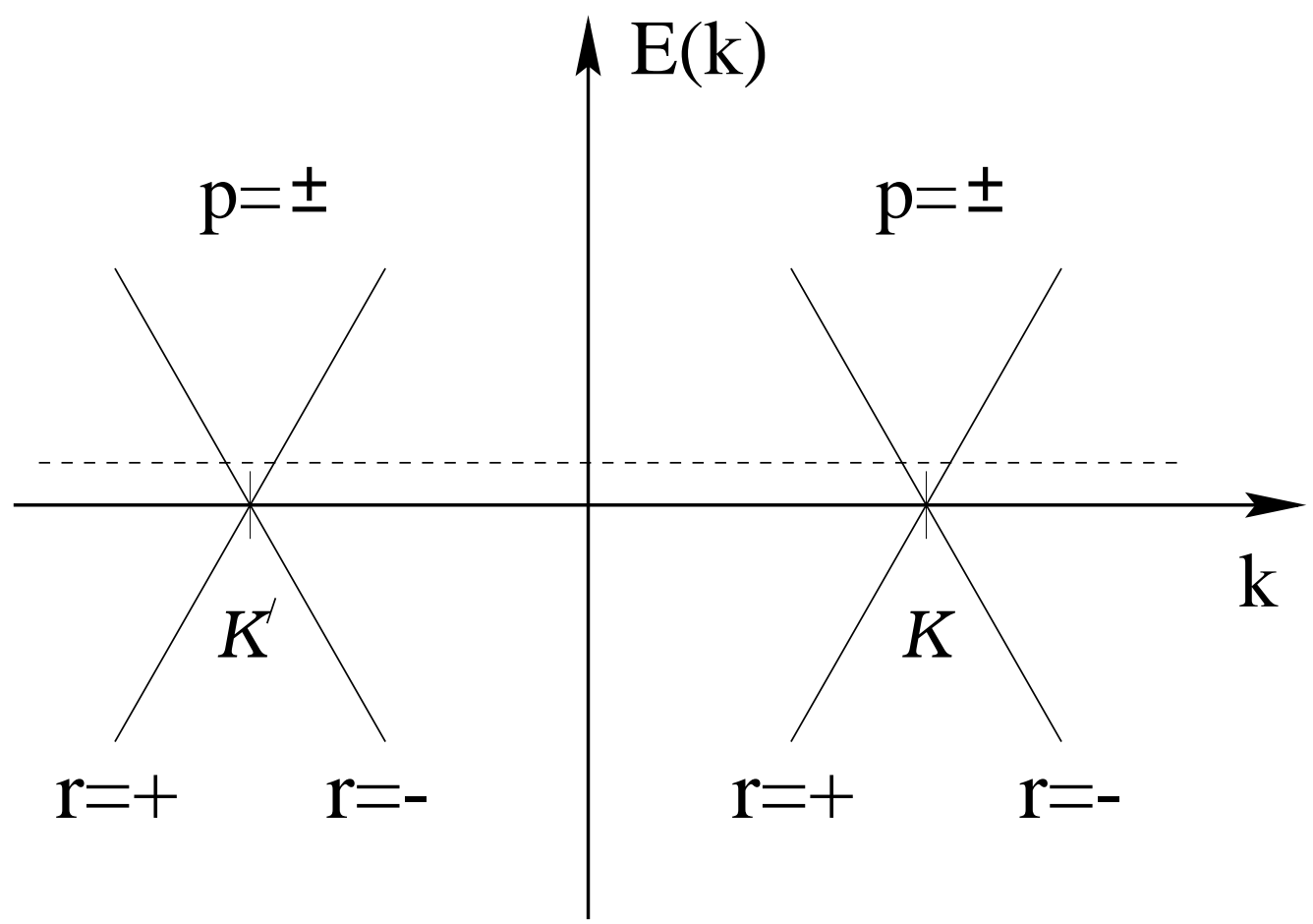

FIG. 1. Schematic bandstructure of a metallic SWNT. A right- and left-moving branch $(r= \pm)$ is found near each of the two Fermi points $\alpha= \pm$ corresponding to $K$ and $K^{\prime}$, respectively. Right- and left-movers arise as linear combinations of the sublattices $p= \pm$. The Fermi energy (dashed line) can be tuned by an external gate. 
(a) $\alpha \mathrm{FS}$

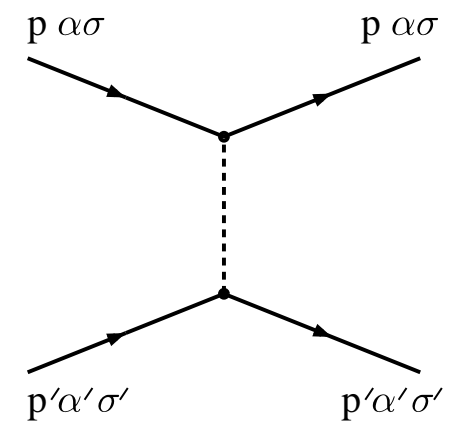

(b) $\alpha \mathrm{BS}$

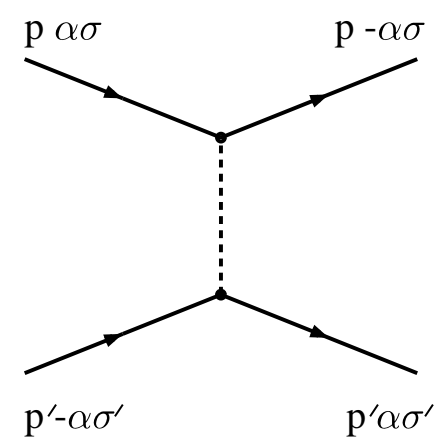

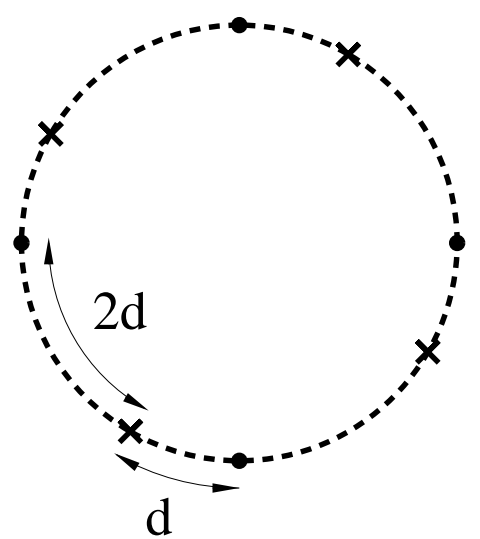

FIG. 3. Microscopic arrangement of carbon atoms around the waist of an armchair SWNT (shown for $n=4$ ). Circles and crosses denote the two distinct sublattices $p= \pm$, and $d=a / \sqrt{3}$.
FIG. 2. Allowed interaction processes away from half-filling. (a) Forward scattering ( $\alpha$ FS). The incoming 1D fermions do not change their quantum numbers $p, \alpha, \sigma$ during the scattering event. (b) Backward scattering ( $\alpha \mathrm{BS})$. One 1D fermion is scattered from Fermi point $\alpha$ to the opposite Fermi point $-\alpha$, with the other being scattered from $-\alpha$ to $\alpha$. Straight lines denote 1D fermion propagators, dashed lines the effective $1 \mathrm{D}$ Coulomb interaction. 

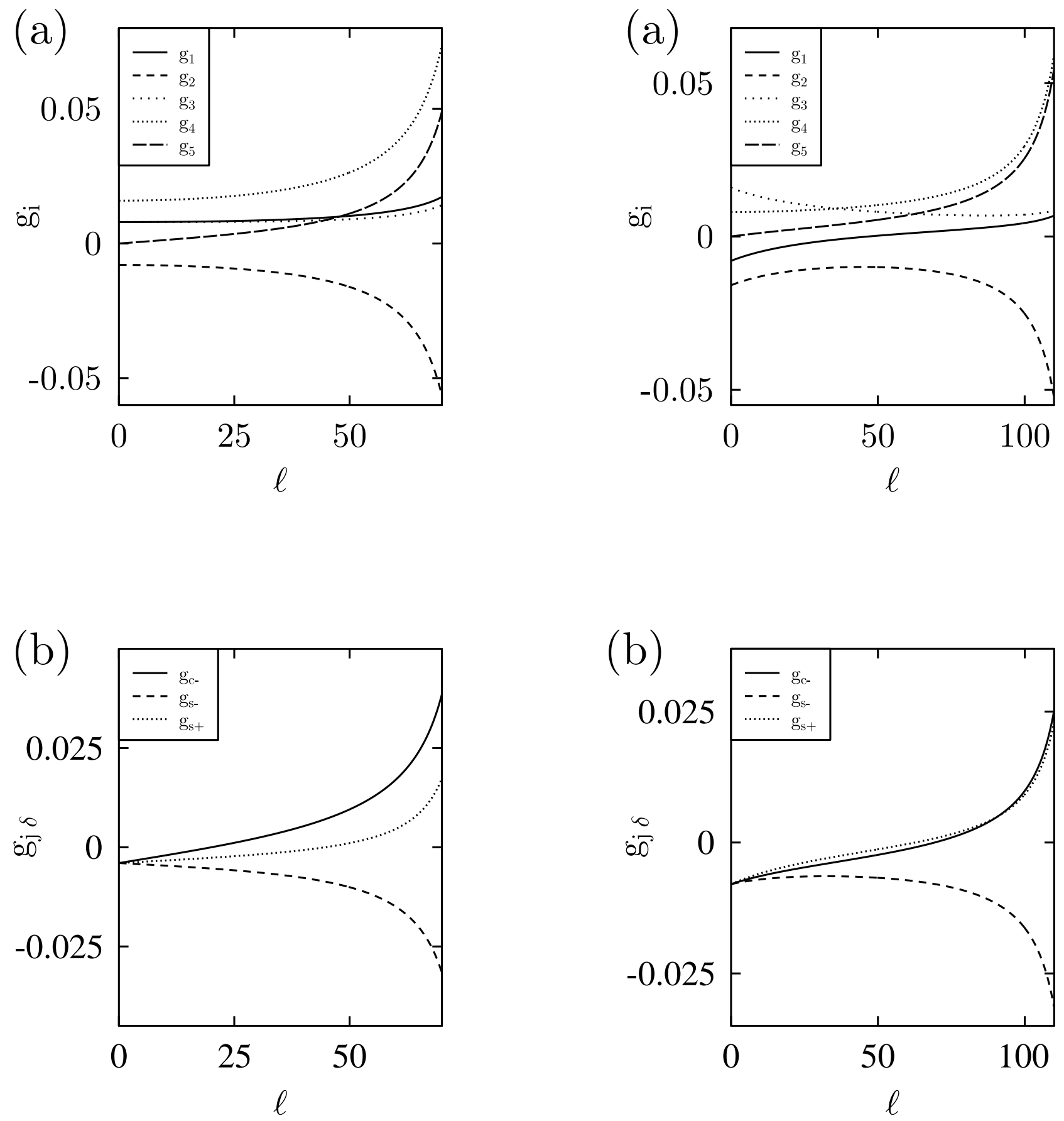

FIG. 4. RG flow of the coupling constants for $f=0.025$ and $b=0.05$. Units are such that $a=1$. Following Eqs. (2.25) and (2.29), these initial values apply to a $(10,10)$ SWNT with externally unscreened Coulomb interactions. 